\title{
Probing the Coherence of Solid-State Qubits at Avoided Crossings
}

\author{
Mykyta Onizhuk $\odot,{ }^{1,2}$ Kevin C. Miao $\odot,{ }^{2}$ Joseph P. Blanton $\odot,{ }^{3,2} \mathrm{He} \mathrm{Ma} \odot,{ }^{1,2}$ \\ Christopher P. Anderson $\odot,{ }^{2}$ Alexandre Bourassa $\odot,{ }^{2}$ David D. Awschalom $\odot,{ }^{3,2,4}$ and \\ Giulia Galli@ 1,2,4,* \\ ${ }^{1}$ Department of Chemistry, University of Chicago, Chicago, Illinois 60637, USA \\ ${ }^{2}$ Pritzker School of Molecular Engineering, University of Chicago, Chicago, Illinois 60637, USA \\ ${ }^{3}$ Department of Physics, University of Chicago, Chicago, Illinois 60637, USA \\ ${ }^{4}$ Materials Science Division and Center for Molecular Engineering, Argonne National Laboratory, Lemont, \\ Illinois 60439, USA
}

(Received 6 October 2020; accepted 23 December 2020; published 20 January 2021)

\begin{abstract}
Optically addressable paramagnetic defects in wide-band-gap semiconductors are promising platforms for quantum communications and sensing. The presence of avoided crossings between the electronic levels of these defects can substantially alter their quantum dynamics and be both detrimental and beneficial for quantum information applications. Here we present a joint theoretical and experimental study of the quantum dynamics of paramagnetic defects interacting with a nuclear spin bath at avoided crossings. We find that we can condition the clock transition of the divacancies in $\mathrm{SiC}$ on multiple adjacent nuclear spins states. We suppress the effects of fluctuating charge impurities and demonstrate an increased coherence time at clock transition, which is limited purely by magnetic noise. Our results pave the way to designing single defect quantum devices operating at avoided crossings.
\end{abstract}

DOI: 10.1103/PRXQuantum.2.010311

\section{INTRODUCTION}

In search of solid-state qubits, electron spin defects in wide-band-gap semiconductors have been extensively explored as robust quantum systems offering both long coherence times [1] and optical readout [2] capabilities for quantum information [3] and quantum sensing [4] applications. In order to design optimal qubits, it is critical to understand and control the interaction between the central electronic spin and the nuclear spin bath. The latter determines, at least in part, the coherence time of qubits, as observed in many solid-state color centers [5-8], but it also offers a platform for robust multiqubit registers for the development of quantum networks [9-12].

The description of the interaction between a central spin and the nuclear bath can be particularly challenging when avoided crossings between energy levels of the central spin occur. Avoided crossings give rise to regimes that can be both beneficial and deleterious to the qubit's coherence. For example, operating at the minimum of the spin transition frequency can significantly increase the

\footnotetext{
*gagalli@uchicago.edu

Published by the American Physical Society under the terms of the Creative Commons Attribution 4.0 International license. Further distribution of this work must maintain attribution to the author(s) and the published article's title, journal citation, and DOI.
}

coherence time when clock transitions arise [13-15]. On the other hand, spin ground-state level anticrossings may lead to an undesired increase in longitudinal relaxation rates $[16,17]$ of spin defects.

Here we investigate the adiabatic dynamics of a qubit interacting with nuclear baths at clock transitions and near ground-state level anticrossings (GSLACs) both theoretically and experimentally. Furthermore, we suggest ways to design and optimize the electron-nuclear spin interactions in clock-transition-based quantum devices.

We focus on neutral divacancies $\left(\mathrm{V}_{\mathrm{C}} \mathrm{V}_{\mathrm{Si}}\right)$ in silicon carbide $(\mathrm{SiC})$, which are promising spin qubit candidates [18-21]. In particular, the axial divacancy [Fig. 1(b)] is one of the most commonly studied defect qubits in $\mathrm{SiC}[22,23]$, with purely axial zero-field splitting. The basal divacancy [Fig. 1(c)], on the other hand, exhibits both an axial and transverse crystal field splitting component, giving rise to clock transitions at zero magnetic field $[15,24]$. We validate our predictions, obtained using the cluster-correlation expansion (CCE) technique, by carrying out Ramsey and Hahn-echo experiments on the basal divacancy, as well as by comparing our theoretical results to previous measurements [5]. Using theory and experiment, we show that in the presence of strongly coupled nuclear spins, multiple clock transitions conditioned on the nuclear spin state may occur. Importantly, we identify the dominant causes of decoherence at avoided crossings and clock transitions, and we discuss the nature of the noise as a function of 

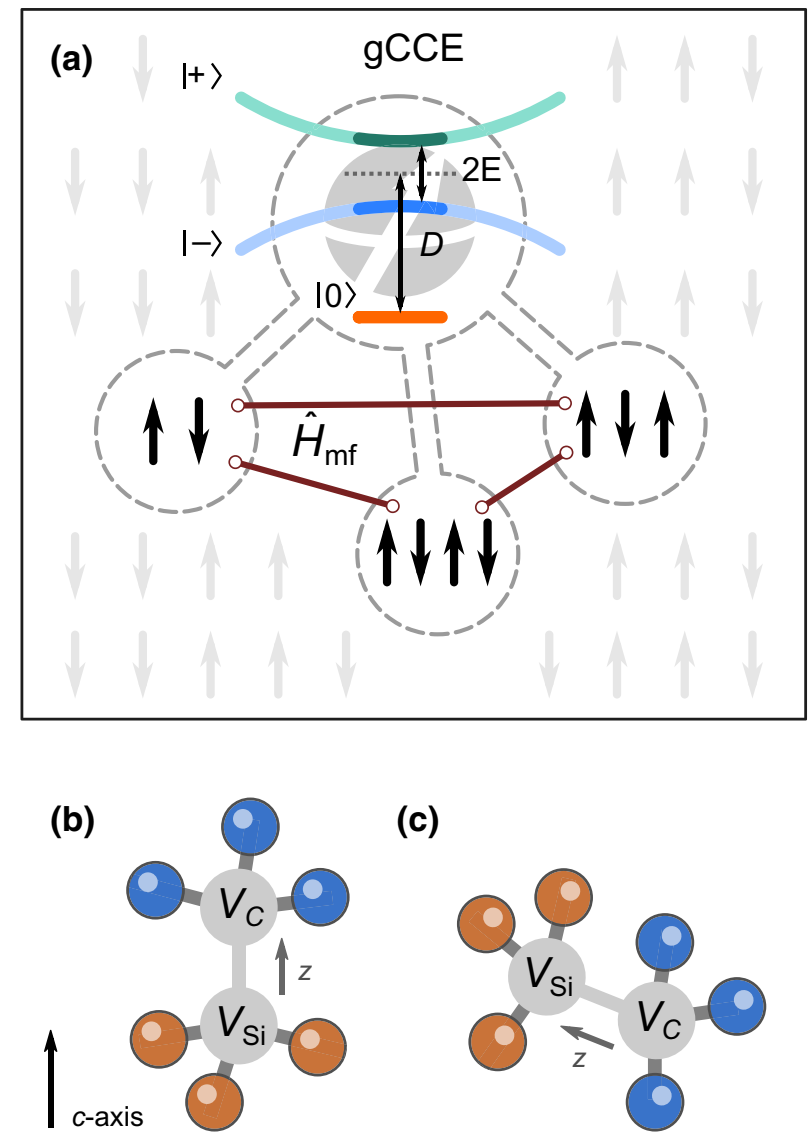

(c)

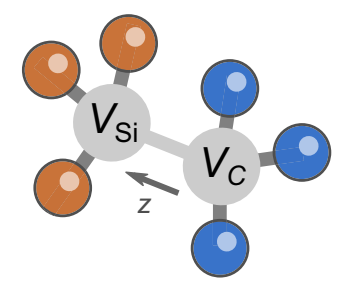

FIG. 1. (a) Schematic representation of the cluster-correlation expansion method used in this work (denoted as gCCE). Each cluster includes the central spin levels (denoted as $|+\rangle,|-\rangle$, and $|0\rangle$ for spin 1 with nonzero longitudinal $D$ and transverse $E$ zerofield splitting). The interactions between clusters are treated at the mean-field level $\left(\hat{H}_{\mathrm{mf}}\right)$, using Monte Carlo sampling of bath states. (b) Schematic representation of the axial $k k$ divacancy. (c) Schematic representation of the basal $k h$ divacancy.

the magnetic field. Finally, we show experimentally that the impact of the nuclear bath on the qubit dynamics can be isolated by employing a charge depletion technique, which leads to an increase of spin coherence time of clock transition qubits by suppressing electric noise.

The rest of the paper is organized as follows. In Sec. II we summarize the theoretical approach used in this work. Next, we discuss the decoherence of axial divacancy near GSLAC in Sec. III. Section IV contains the Ramsey and Hahn-echo predictions and measurements for basal divacancy near clock transition. We describe a change in nature of the noise near avoided crossings in Sec. V, and in Sec. VI we discuss further ways to engineer coherence protection. Finally, we conclude the paper in Sec. VII, discussing the general implications of our findings.

\section{THEORETICAL FRAMEWORK}

\section{A. Spin defect Hamiltonian}

We investigate the dynamics of a spin qubit by studying the evolution of the central spin interacting with the nuclear spin bath. The Hamiltonian of a given system in an external magnetic field can be written as a sum of the central spin Hamiltonian $\hat{H}_{e}$, and nuclear Zeeman splitting, hyperfine coupling, and nuclear dipolar coupling terms:

$$
\hat{H}=\hat{H}_{e}-\sum_{i} \gamma_{n, i} B_{z} \hat{I}_{z, i}+\sum_{i} \mathbf{S A} \mathbf{I}_{i}+\sum_{i \neq j} \mathbf{I}_{i} \mathbf{P} \mathbf{I}_{j} .
$$

Here $B_{z}$ is the magnetic field oriented along the $z$ axis, $\gamma_{n, i}$ is the gyromagnetic ratio of the $i$ th nuclear spin, $\mathbf{S}=$ $\left(\hat{S}_{x}, \hat{S}_{y}, \hat{S}_{z}\right)$ and $\mathbf{I}_{i}=\left(\hat{I}_{x, i}, \hat{I}_{y, i}, \hat{I}_{z, i}\right)$ denote electron and the $i$ th nuclear spin operators, respectively, $\mathbf{A}_{i}$ is the hyperfine coupling tensor of the $i$ th nuclear spin, and $\mathbf{P}_{i j}$ is the dipole-dipole coupling between spins $i$ and $j$.

The electron Hamiltonian $\hat{H}_{e}$ includes the Zeeman interaction with the external magnetic field and a zero-field splitting (ZFS) term with longitudinal $(D)$ and transverse (E) components:

$$
\begin{gathered}
\hat{H}_{e}=-\gamma_{e} B_{z} \hat{S}_{z}+D\left[\hat{S}_{z}^{2}-\frac{1}{3} S(S+1)\right]+E\left(\hat{S}_{x}^{2}-\hat{S}_{y}^{2}\right) . \\
\text { B. Cluster-correlation expansion method for } \\
\text { avoided crossings }
\end{gathered}
$$

The CCE method is one of the leading approaches for the simulation of quantum dynamics of spin qubits interacting with a multitude of bath spins [5,25-27]. The method approximates the off-diagonal elements of the qubit density matrix as a product of contributions from independent nuclear spin clusters. Recently, the CCE technique was successfully applied to study clock transitions of bismuth spin qubits in silicon (Si:Bi) with an explicit diagonalization of central spin energy levels within each cluster [28,29].

In a similar fashion, in this work we adopt the generalized CCE (gCCE) formulation [30] to compute both the population change and coherence of the central spin at avoided crossings [Fig. 1(a)]. The generalized CCE is distinct from the "pseudospin" model conventionally used in CCE calculations $[5,26,31,32]$ as it includes the full central spin Hamiltonian in each cluster. In particular, here we compute elements of the density matrix of the central spin $\rho_{a b}=\langle a|\hat{\rho}| b\rangle$ as the product of cluster contributions, where $a, b$ denote different spin states (e.g., $m_{s}=1,0,-1$ states for spin-1 systems):

$$
\rho_{a b}=\tilde{\rho}_{a b}^{\{0\}} \prod_{i} \tilde{\rho}_{a b}^{\{i\}} \prod_{i, j} \tilde{\rho}_{a b}^{\{i j\}} \cdots
$$

Here $\{0\}$ denotes a cluster consisting of a free central spin, $\{i\}$ a cluster including the central spin and nuclear spin $i$, 
and so on. The maximum size of the cluster in Eq. (3) defines the order of the approximation. For example, at first order (gCCE1), only isolated nuclear spins $\left(\rho^{\{i\}}\right)$ are included in the expansion. At second order (gCCE2), contributions from pairs of nuclear spins $\left(\rho^{\{i, j\}}\right)$ are added, etc.

We note that at clock transitions previously studied in the Si:Bi system [28], the large splitting at avoided crossings induced by the interaction between electron spin and Bi nuclear spin leads to a pure dephasing regime of the qubit decoherence. Balian et al. [28] established that in such systems the inhomogeneous magnetic noise can be treated macroscopically. Instead, for qubits with small energy splittings at zero applied magnetic field, we find that a careful microscopic treatment of Overhauser fields is required for each spatial configuration of nuclear spins, consistent with the conclusion of the recent work by Zhang et al. [33] (see Appendix A).

We therefore perform calculations with randomly sampled pure states of the spin bath, and for each pure state, we include the mean-field effect of all the nuclear spins outside a chosen cluster $\hat{H}_{\mathrm{mf}}$ [Fig. 1(a)]. Such treatment also allows for different energy splittings of spins within a cluster, improving the numerical convergence of cluster dynamic simulations [34]. Further details are available in Appendix B.

\section{DECOHERENCE AT GROUND-STATE LEVEL ANTICROSSINGS}

We start by investigating the dynamics of the axial $k k-\mathrm{V}_{\mathrm{C}} \mathrm{V}_{\mathrm{Si}}$ defect in $4 H-\mathrm{SiC}$ near its GSLAC. The measured ensemble-averaged Hahn-echo coherence times $\left(T_{2}\right)$ of this defect reaches $1.3 \mathrm{~ms}$ in samples with natural isotopic concentration [5]. Because of its $C_{3 v}$ symmetry, the ZFS entering Eq. (2) has only a longitudinal component $D=1.305 \mathrm{GHz}$ [35], and the qubit levels may be chosen as the $\left|-1_{z}\right\rangle$ and $\left|0_{z}\right\rangle$ eigenstates of $S_{z}$, where $z$ is the spin quantization axis.

The coherence time of the $k k-\mathrm{V}_{\mathrm{C}} \mathrm{V}_{\mathrm{Si}}$ divacancy was successfully predicted with the conventional CCE method for a wide range of magnetic fields, and the homonuclear pair-wise spin flips were found to be the main source of decoherence at strong fields [5]. However, a significant decrease in the coherence time is observed in experiments when the magnetic field approaches approximately $45 \mathrm{mT}$; such a decrease is not captured by conventional CCE calculations with a "pseudospin" model [Fig. 2(a)], suggesting a decoherence mechanism beyond pure dephasing.

We perform gCCE calculations with Monte Carlo sampling of bath states [Fig. 2(d)] and we correctly obtain a local minimum in $T_{2}(B)$ for $B_{z}=46.6 \mathrm{mT}$, while reproducing the results of conventional CCE calculations for other values of $B_{z}$. We note that, while previous CCE results [5]
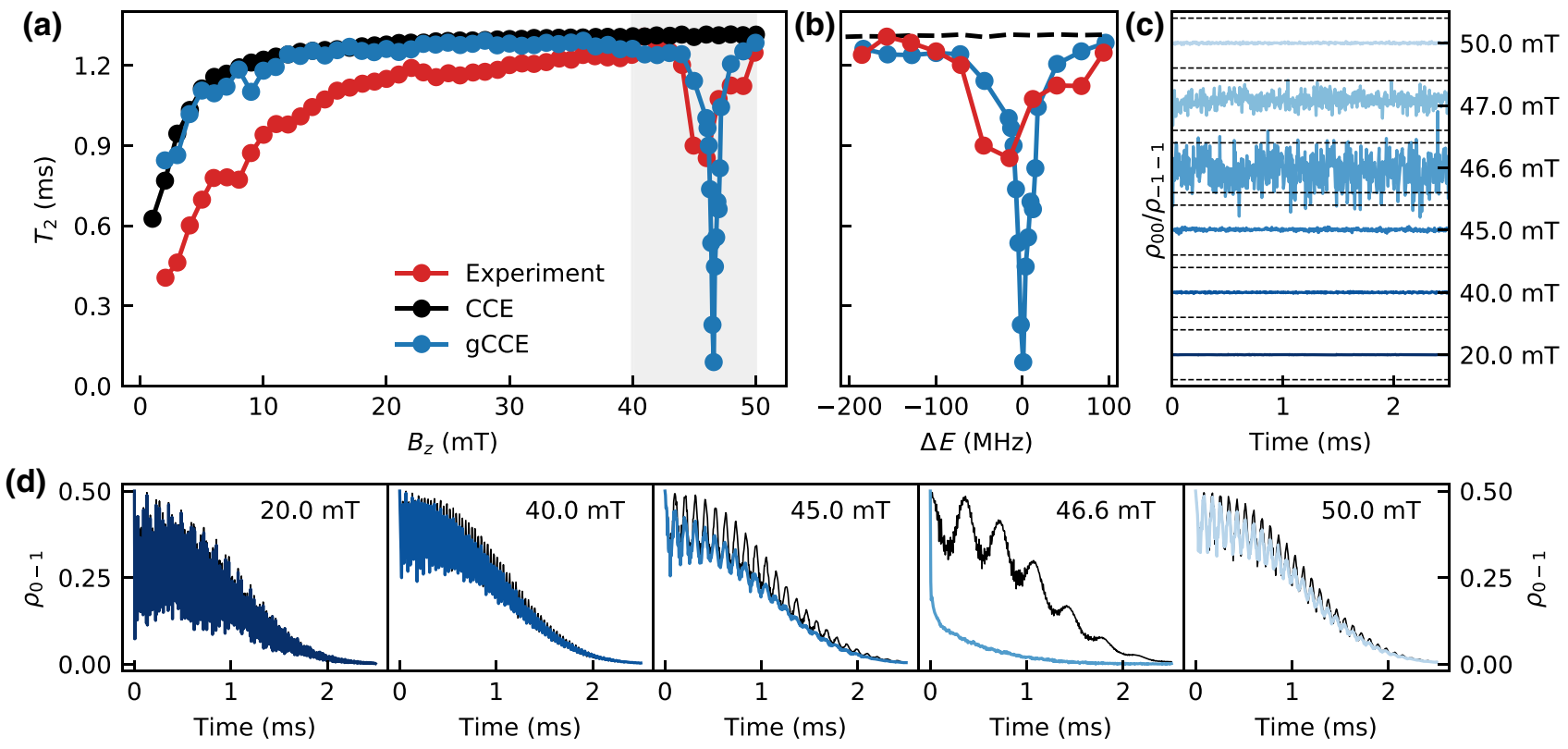

FIG. 2. Ensemble Hahn-echo coherence of the axial $k k$ divacancy in $4 H$-SiC. (a) Coherence time $T_{2}$ (blue circles) as a function of the magnetic field $B_{z}$. The experimental results (red circles) and conventional CCE predictions (black circles) are from Ref. [5]. Gray shading denotes the range of $T_{2}$ shown in (b). (b) Experimental (red circles) and predicted (blue circles) coherence times $T_{2}$ as a function of energy splitting between qubit levels. The black line shows the pure dephasing results. (c) The oscillations in the ratio of the diagonal elements of the density matrix of the divacancy as a function of time for various values of the magnetic field. The dashed lines show the $\pm 2 \%$ range. (d) The off-diagonal elements of the density matrix for different magnetic fields computed using the gCCE method with Monte Carlo sampling of the bath states (color) and using the conventional CCE method (black). 
were obtained using a point dipole approximation, here we use accurate hyperfine couplings predicted by ab initio calculations (see Appendix C). This difference in hyperfine couplings accounts for the small discrepancy between CCE and gCCE results observed at small fields.

The origin of the minimum in $T_{2}(B)$ can be understood by analyzing the populations of different spin levels [Fig. 2(c)]. For most values of $B$, the population of the electron spin levels is constant. However, there are values of $B$ for which the energy difference between the $|1\rangle$ and $|0\rangle$ levels is of the same order of magnitude as the hyperfine interaction with nuclear spins. We note that, when the energy splitting falls below approximately $100 \mathrm{MHz}$, significant deviations from pure dephasing occur [Fig. 2(b)]. In this case, the electron spin experiences large population fluctuations that lead to a significant decrease in the coherence time near the GSLAC. Therefore, we conclude that at a GSLAC, the longitudinal relaxation process substantially contributes to decreasing the Hahn-echo coherence time, as observed experimentally.

\section{DECOHERENCE AT CLOCK TRANSITIONS}

As mentioned earlier, the basal divacancy $k h-\mathrm{V}_{\mathrm{C}} \mathrm{V}_{\mathrm{Si}}$ exhibits a clock transition at zero magnetic field, which arises from ZFS interactions, unlike the one in the $\mathrm{Bi}: \mathrm{Si}$ donor qubits studied in Refs. [29,32]. The $k h-\mathrm{V}_{\mathrm{C}} \mathrm{V}_{\mathrm{Si}}$ defect has $C_{1 h}$ symmetry, leading to a nonzero transverse component of the ZFS: $E=18.4 \mathrm{MHz}$ [24]. Combined with a strong longitudinal splitting $(D=1.334 \mathrm{GHz})$, the ZFS tensor leads to an avoided crossing of electron spin levels at zero magnetic field from which a clock transition emerges. The qubit levels at the clock transition correspond to $|+\rangle=(1 / \sqrt{2})\left(\left|1_{z}\right\rangle+\left|-1_{z}\right\rangle\right)$ and $|0\rangle=\left|0_{z}\right\rangle$. The frequency of clock transitions is insensitive to magnetic fields to first order, thus increasing protection from the nuclear bath induced decoherence [14].

\section{A. Impact of nuclear spin coupling}

In order to understand how the coupling strength between the central spin and the nuclear spins affects clock transitions, we experimentally investigate three different single $k h-\mathrm{V}_{\mathrm{C}} \mathrm{V}_{\mathrm{Si}}$ divacancy qubits (see Appendix $\mathrm{E}$ for the experimental procedure). They are labeled VVA, VVB, and $\mathrm{VVC}$, and they represent configurations with weakly (VVA) and strongly interacting (VVB and VVC) nuclear spins. We obtain theoretical configurations directly comparable with the experimental ones by generating a set of random nuclear spin configurations in the $\mathrm{SiC}$ lattice with the same number of strongly interacting nuclear spins as observed in the measured Ramsey fringes. Out of this set, we then select the configurations with a computed value of $T_{2}^{*}$ at $B_{z}=50 \mu \mathrm{T}$ similar to the measured one (see Appendix D for details).
We first analyze the VVA configuration, which contains only weakly coupled nuclear spins. Its frequency spectrum, obtained as a Fourier transform of Ramsey fringe oscillations, can be simply represented by one hyperbola [Figs. 3(a) and 3(b)]. In the absence of a nuclear bath, the frequency of the clock transition is given by

$$
\omega-\omega_{0}=\sqrt{\gamma_{e}^{2} B_{z}^{2}+E^{2}} .
$$

We obtain good agreement between theoretical predictions and the measured Ramsey fringes at small fields, but in the zero-field regime the experimentally observed decoherence is significantly faster [Fig. 3(h)]. We find that this apparent discrepancy is due to the electric noise affecting the qubit state, as we explain below.

When operated near a clock transition, the basal divacancy spin becomes first-order insensitive to magnetic fluctuations. However, a first-order sensitivity to electric field fluctuations emerges, due to the linear dependence of the ZFS tensor components on the local electric field [36]. Therefore, the electron spin dephasing time becomes limited by the electric field noise $[12,15,36]$. In $\mathrm{SiC}$ divacancies, the electric noise is primarily caused by charge state fluctuations of photoactive impurities, which may undergo charge state transitions under optical excitation $[37,38]$, leading to a variation of local electric fields.

In our experiments, we use charge depletion [24,37] to deactivate photoactive impurities within the optical excitation region, thus substantially reducing the electric field contributions to the ground-state spin dephasing. We apply $13 \mathrm{~V}$ across a lithographically patterned capacitor with a $10 \mu \mathrm{m}$ gap width. The applied electric field acting on a divacancy located between the capacitor plates ionizes the undesired charge carriers and removes them from photoactive impurities in the proximity of the divacancy. This technique allows us to isolate the contributions of the magnetic field noise near the clock transition [Fig. 3(g)] and to perform a meaningful comparison with our theoretical model.

We find that, under charge depleted conditions, the measured coherence time is substantially increased and the experimentally observed Ramsey precession at zero field agrees well with the theoretical prediction of the gCCE. Interestingly, in the presence of a weak magnetic field, an agreement between theory and experiment is obtained without applying any charge depletion, indicating that the decoherence rate in this case is not limited by electric noise.

We now turn to lattice configurations with strongly coupled nuclear spins. We first consider the defect labeled VVB, for which we observe a splitting in the frequency spectrum due to the presence of one strongly coupled nuclear spin [Figs. 3(c) and 3(d)]. Each of the hyperbolae shown in the figure corresponds to the oscillation 


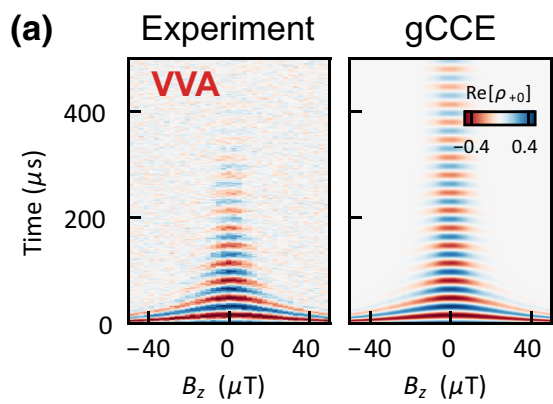

(c)

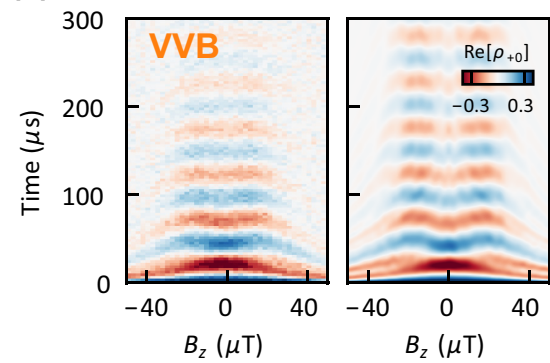

(e)

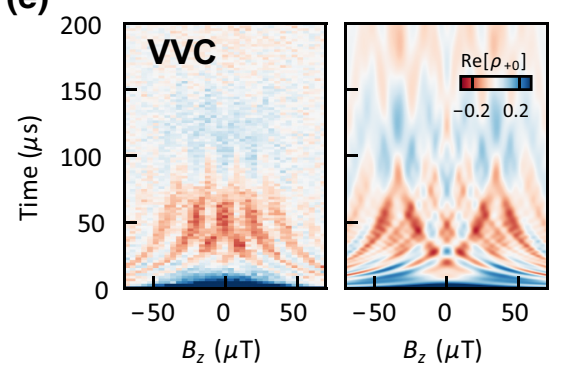

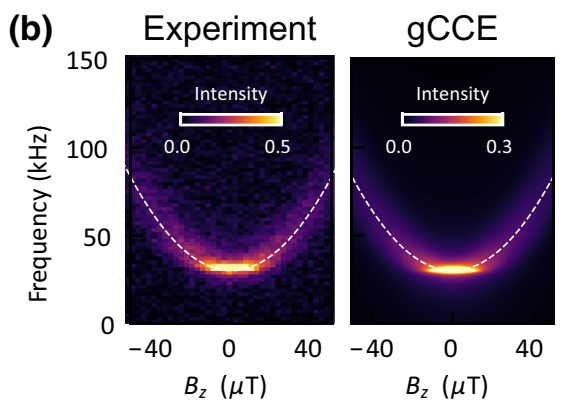

(d)

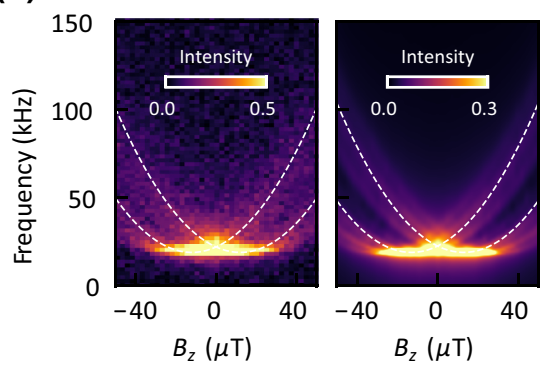

(f)

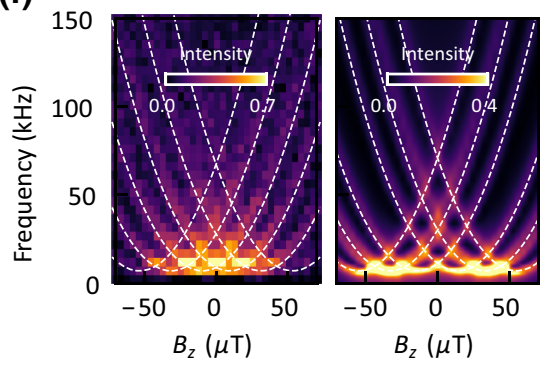

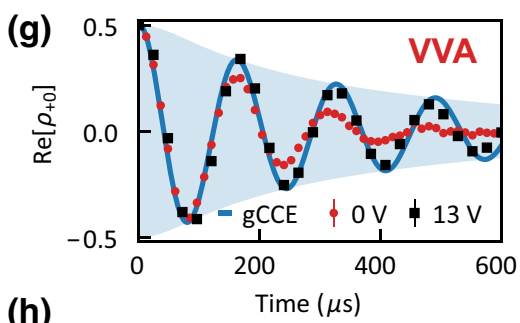

(h)
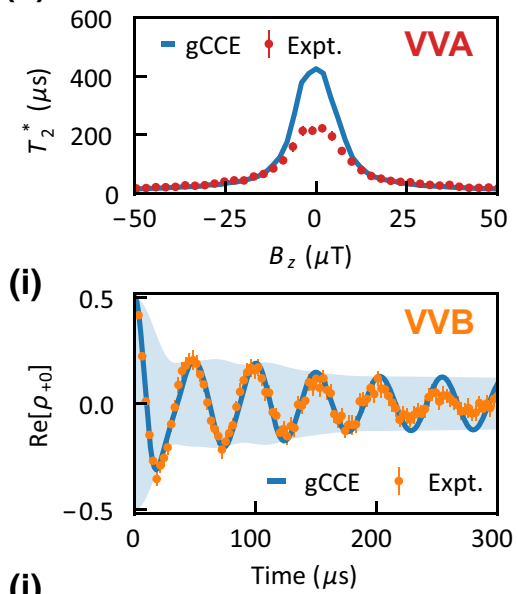

(j)

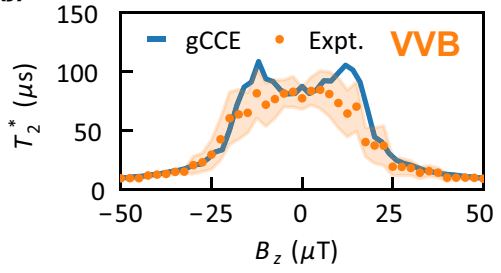

FIG. 3. Ramsey interferometry for three experimental $k h-V_{C} V_{S i}$ systems. (a)-(f) Ramsey precession and frequency spectrum for a defect (a),(b) with only weakly coupled nuclear spins (VVA), (c),(d) with one strongly coupled nuclear spin (VVB), and (e),(f) with three strongly coupled nuclear spins (VVC). For each defect, we show theoretical predictions and experimental results. White dashed lines show the positions of the hyperbolae [see Eq. (4)]. (g) Measured Ramsey precession of VVA at zero field with (black) or without (red) charge depletion (see the text), compared to the theoretical prediction (blue). The shaded area corresponds to the theoretically predicted decay. (h),(j) Distribution of $T_{2}^{*}$ for VVA (h) and VVB (j) as a function of the magnetic field $\left(B_{z}\right)$. Shaded area in (j) shows the error of the fit. (i) Measured Ramsey precession of VVB at weak applied magnetic field $\left(B_{z}=12.5 \mu \mathrm{T}\right)$ compared to the theoretical prediction. Error bars correspond to 2SD.

frequency of Ramsey fringes of the divacancy, coupled to either the spin-up or spin-down nuclear state. The minimum of each hyperbola occurs when the magnetic field is equal to the hyperfine field of the strongly coupled nuclear spin, $\left|A_{i z} / 2 \gamma_{e}\right|=\left|B_{z}\right|$, where $A_{i z}=\sqrt{A_{x z}^{2}+A_{y z}^{2}+A_{z z}^{2}}$ [24]. By solving this equation, we obtain the hyperfine parameter of the strongly coupled nuclear spin in VVB: $A_{i z} \approx 0.6 \mathrm{MHz}$.

In Figs. 3(c) and 3(d) we compare with experiments our theoretical results for a nuclear configuration for which the computed $A_{i z}$ is $0.75 \mathrm{MHz}$. We find excellent agreement for the time evolution and the frequency spectrum. We note that, due to the presence of the strongly coupled spin, the Ramsey precession exhibits a fast and a slow decay mode [Fig. 3(i)], and the full dynamics of the decoherence process may not be described by a single $T_{2}^{*}$ [Fig. 3(j)].
However, by initializing the strongly coupled nuclear spin so that it is antiparallel to the external magnetic field, one can eliminate the fast decay mode, and, together with the charge depletion strategy outlined above, one may achieve a substantial increase (by a factor of 5) in the coherence time (see Appendix D).

In the presence of several strongly coupled nuclear spins, further splitting of the frequency spectrum may occur. In this case, the minimum of each hyperbola is located at

$$
B_{z}=\sum_{\text {strong }} \pm \frac{A_{i z}}{2 \gamma_{e}} .
$$

The measured frequency spectrum of the defect labeled VVC contains six separate hyperbolae, suggesting the presence of three strongly coupled nuclear spins with two 

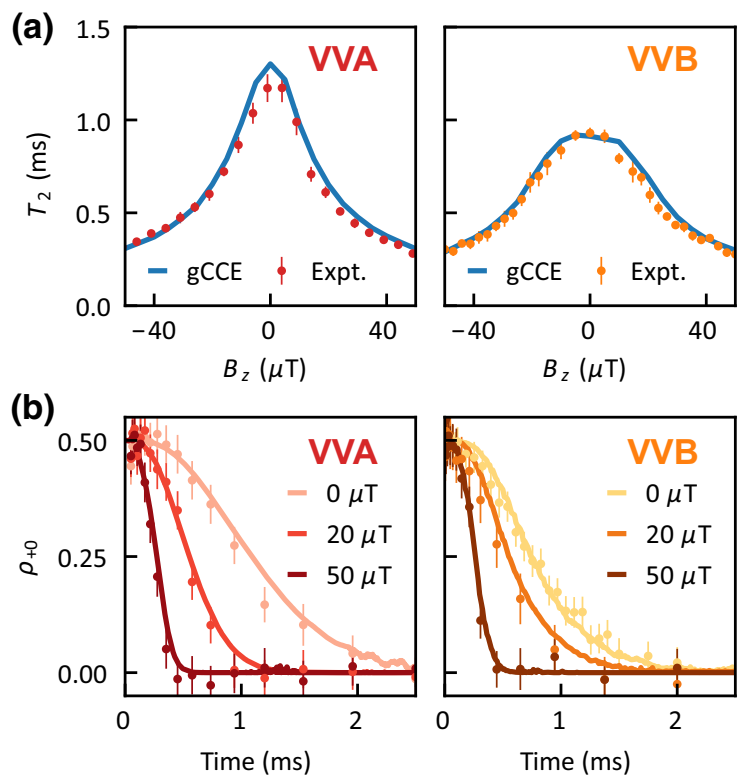

FIG. 4. Single defect Hahn-echo coherence time of the $k h$ $\mathrm{V}_{\mathrm{C}} \mathrm{V}_{\mathrm{Si}}$ divacancy. (a) Distribution of $T_{2}$ for VVA (left) and VVB (right) as a function of the magnetic field $B_{z}$ (VVA and VVB are the same defects represented in Fig. 3). (b) The Hahn echo decay for three different values of the magnetic field. Solid lines correspond to theoretical predictions, points to experimental measurements. Error bars correspond to 2SD.

of them having similar hyperfine parameters [Figs. 3(e) and 3(f)]. We identify one nuclear spin with $A_{i z} \approx 1.7$ $\mathrm{MHz}$ and two nuclear spins with $A_{i z} \approx 0.6 \mathrm{MHz}$. Hence, we compare theory and experiment using calculations for a nuclear configuration that contains three strongly coupled nuclear spins with similar values of the hyperfine constants: $A_{i z}=1.92,0.65,0.49 \mathrm{MHz}$. We obtain good agreement in both the time and frequency domains. However, due to the complexity of the dynamics, a simple exponential decay cannot reliably characterize the decoherence time of VVC; nevertheless, the decoherence occurs on a timescale of $200-300 \mu \mathrm{s}$, both in theory and experiment.

Furthermore, we carry out a study of the Hahn-echo decoherence time for the VVA and VVB defects [Figs. 4(a) and 4(b)] and again find excellent agreement between experimental values and theoretical predictions. In VVB, the presence of the strongly coupled nuclear spin leads to a broadening of the coherence time peak compared to VVA, and to a decrease in the maximum of $T_{2}$ [0.930(14) $\mathrm{ms}$ for VVB versus 1.17(4) ms for VVA]. We note that the measured and computed zero-field Hahn-echo coherence times agree even without applying any charge depletion to the sample, suggesting that the electric noise has a minor impact on $T_{2}$.

\section{NATURE OF NUCLEAR NOISE IN SOLID-STATE QUBITS}

Having validated the predictions of the gCCE with several experiments, we can now analyze the nature of the nuclear noise in the decoherence processes of the $k h-\mathrm{V}_{\mathrm{C}} \mathrm{V}_{\mathrm{Si}}$ divacancy.

We compute the coherence time of the $k h-\mathrm{V}_{\mathrm{C}} \mathrm{V}_{\mathrm{Si}}$ divacancy at zero field $(0 \mathrm{~T})$, where a clock transition occurs, and at $B_{z}=0.1 \mathrm{~T}$ where we expect the basal and axial divacancies to exhibit similar coherence properties. We consider eigenstates of $\hat{S}_{z}$ as qubit levels at $0.1 \mathrm{~T}$. We find that at both zero and strong magnetic fields, the Ramsey decay is limited by static thermal noise arising from the entanglement of the qubit with pure states of the bath, which remain unchanged in time [27]:

$$
\hat{\rho}(0) \otimes \sum_{B} p_{B}|B\rangle\left\langle B\left|\rightarrow \sum_{B} p_{B} \hat{\rho}_{B}(t) \otimes\right| B\right\rangle\langle B| .
$$

Indeed, our calculations of $T_{2}^{*}$ for the $k h-\mathrm{V}_{\mathrm{C}} \mathrm{V}_{\mathrm{Si}}$ divacancy in the weakly coupled bath [Figs. 5(a) and 5(b)] show that the inhomogeneous coherence time depends on the hyperfine parameters only through the average bath coupling $\left(\sqrt{\sum_{i} A_{i z}^{2}}\right)$, as expected in the case of static thermal noise [40].
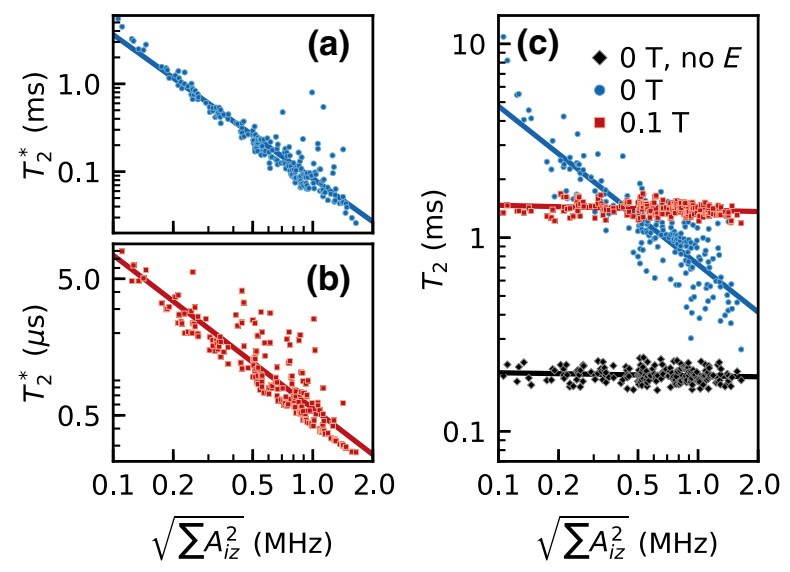

FIG. 5. Single defect coherence times $T_{2}^{*}$ and $T_{2}$ of the $k h$ $\mathrm{V}_{\mathrm{C}} \mathrm{V}_{\mathrm{Si}}$ divacancy at zero and high magnetic fields for different bath couplings. (a),(b) The coherence time $T_{2}^{*}$ for zero (a) and high (b) fields as a function of the square root of the sum of squares of the hyperfine couplings with bath spins $\sqrt{\sum_{i} A_{i z}^{2}}$. Significant deviations from the least-squares fit (solid lines) are present for systems containing single nuclear spins with high hyperfine coupling, and the coherence decay may not be approximated by a single exponential [39]. (c) The coherence time $T_{2}$ for zero (blue) and high (red) magnetic fields, and for a hypothetical system with $E=0 \mathrm{MHz}$ at zero field (black). All calculations are performed for $\mathrm{SiC}$ with natural isotope concentration for the weakly coupled nuclear bath. 
The nature of noise is different in Hahn-echo experiments, where the $\pi$ pulse removes the static part of the noise dominating the Ramsey decay, and $T_{2}$ depends only on the dynamical fluctuations of the magnetic field due to nuclear spin flips [27]. If the flips are completely random, the decay rates originate primarily from the accumulation of random phases due to dynamical fluctuations; in this case the noise is by definition classical and the variance of the noise distribution is given by $\sum_{i} A_{i z}^{2}$ [41]. Therefore, we expect the coherence time to vary linearly on a logarithmic scale as a function of $\sqrt{\sum_{i} A_{i z}^{2}}$ in systems where the noise is classical [42]. On the other hand, when the back action of the central spin is dominant (i.e., the dynamics of the nuclear bath is strongly influenced by the electron spin state [27]), the coherence dynamics deviates from that predicted using classical approximations [43]. In Fig. 5(c) we show $T_{2}$ of the $k k-\mathrm{V}_{\mathrm{C}} \mathrm{V}_{\mathrm{Si}}$ divacancy as a function of $\sqrt{\sum_{i} A_{i z}^{2}}$ in the zero and strong field regimes. At zero field, $T_{2}$ varies as $\sqrt{\sum_{i} A_{i z}^{2}}$, with more than an order of magnitude difference in $\sqrt{\sum_{i} A_{i z}^{2}}$ between the different configurations. This dependence suggests a stochastic nature of the noise at clock transitions and is consistent with the results reported for bismuth qubits in silicon [32]. At strong fields, $T_{2}$ is instead independent of the average coupling to the bath, consistent with the quantum nature of the noise, expected in this regime. These results show that the noise affecting Hahn-echo experiments is different at clock transitions and in the strong field regime, and the transition from classical to quantum noise may be tuned by simply varying the applied magnetic field.

We note that the differences in the nature of the noise is not sufficient to explain why the average value of $T_{2}$ at the clock transition of the basal divacancy is similar to that at a strong field $(1.15 \mathrm{~ms}$ versus $1.4 \mathrm{~ms})$. This similarity arises from the combination of two competing effects: strong electron spin backaction, leading in principle to a reduction in the coherence time, and the Zeeman splitting of nuclear spins, having instead the opposite effect. We can isolate the effect of the electron spin's strong backaction on the coherence time, by considering a hypothetical $k h-\mathrm{V}_{\mathrm{C}} \mathrm{V}_{\mathrm{Si}}$ divacancy with $E=0 \mathrm{MHz}$ under a zero magnetic field [Fig. 5(c), the $0 \mathrm{~T}$, no $E$ case]. We find that $T_{2}$ of this system is independent from $\sqrt{\sum_{i} A_{i z}^{2}}$, and the ensemble average $T_{2}$ is $0.2 \mathrm{~ms}$, significantly smaller than that obtained for the clock transition, confirming that electron backaction is indeed responsible for an increase in the decoherence rate. Under a strong magnetic field, the Zeeman splitting of the nuclear spins is instead responsible for a decrease in decoherence rates. The splitting can be larger than both the interaction strength between nuclear spins and the hyperfine coupling, leading to the suppression of spin nonconserving flips [39]. Only the secular pairwise flip flops of nuclear spins with the same gyromagnetic ratio $(\uparrow \downarrow \leftrightarrow \downarrow \uparrow)$ are possible in this regime [5,44], thus greatly reducing the number of possible spin flips and decreasing the decoherence rate [Fig. 2(a)].

\section{ENGINEERING QUBIT PROTECTION AT A CLOCK TRANSITION}

It is interesting to analyze in detail the effect of the magnitude of the transverse component of the ZFS on coherence protection. To this end, we investigate how coherence times vary as a function of a hypothetical change in $E$ for the $k h-\mathrm{V}_{\mathrm{C}} \mathrm{V}_{\mathrm{Si}}$ divacancy, within a weakly coupled nuclear bath [Figs. 6(a) and 6(b)]. We find that the ensemble averaged coherence time scales sublinearly as a function of the transverse ZFS $\left(T_{2} \approx 0.34 E^{0.43}, T_{2}^{*} \approx 0.03 E^{0.61}\right.$; see Appendix $\mathrm{D}$ for the distribution of single defect coherence times). Our calculations show that defects with large transverse ZFS will exhibit substantially higher protection from magnetic noise.

In particular, we predict the coherence time of the $h k-\mathrm{V}_{\mathrm{C}} \mathrm{V}_{\mathrm{Si}}$ basal divacancy. The $h k-\mathrm{V}_{\mathrm{C}} \mathrm{V}_{\mathrm{Si}}$ divacancy has significantly higher transverse ZFS than the $k h-\mathrm{V}_{\mathrm{C}} \mathrm{V}_{\mathrm{Si}}$ divacancy $(E=82.0 \mathrm{MHz})$, and similar longitudinal ZFS $\left(D=1.222 \mathrm{GHz}\right.$ ) [35]. The total distribution of $T_{2}$ and $T_{2}^{*}$ as a function of the magnetic field for different spatial configurations of the weakly coupled nuclear bath is shown

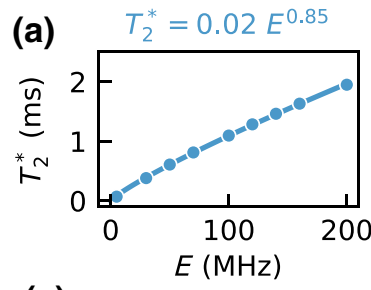

(c)

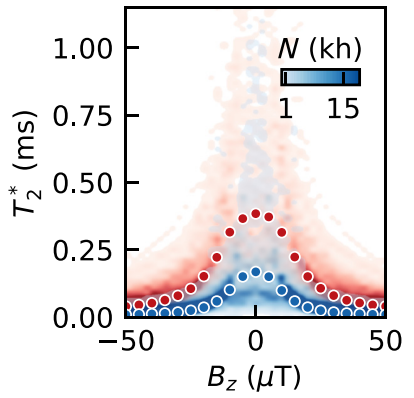

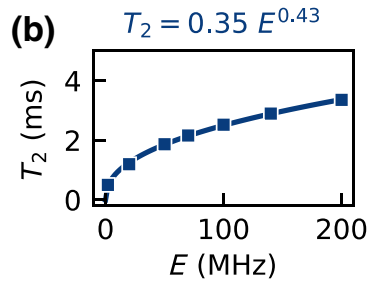

(d)

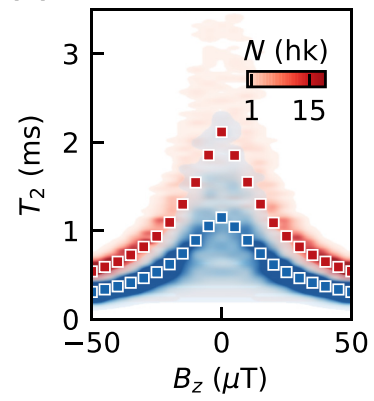

FIG. 6. Dependence of the coherence time on the transverse zero-field splitting. (a),(b) Coherence times $T_{2}^{*}$ (a) and $T_{2}$ (b) of the $k h-\mathrm{V}_{\mathrm{C}} \mathrm{V}_{\mathrm{Si}}$ divacancy with different hypothetical values of transverse ZFS E. (c),(d) The heat map of the single defect $T_{2}^{*}$ $\left(T_{2}\right)$ for 120 different nuclear spin configurations of both the $h k$ $\mathrm{V}_{\mathrm{C}} \mathrm{V}_{\mathrm{Si}}$ (red) and $k h-\mathrm{V}_{\mathrm{C}} \mathrm{V}_{\mathrm{Si}}$ (blue) divacancies as a function of the magnetic field. The color corresponds to the number of configurations with a given $T_{2}^{*}\left(T_{2}\right)$ at the given magnetic field. The circles (squares) indicate the ensemble averaged coherence time. 
in Figs. 6(c) and 6(d) for both basal defects. We can see that there is a significant variability in the value of the coherence time at $B=0$. The increase in the transverse ZFS leads both to a significant increase in the maximum value of the coherence time and to an increased robustness towards the external magnetic field. The ensemble average zero field $T_{2}^{*}=380 \mu$ s of the $h k-\mathrm{V}_{\mathrm{C}} \mathrm{V}_{\mathrm{Si}}$ divacancy is predicted to be 2.3 times higher than that of the $k h-\mathrm{V}_{\mathrm{C}} \mathrm{V}_{\mathrm{Si}}$ divacancy, and the $T_{2}=2.12 \mathrm{~ms}$ is found to be increased by a factor of 1.8, in good agreement with Figs. 6(a) and $6(\mathrm{~b})$. In the presence of a strong field $(0.1 \mathrm{~T})$ the ensemble averaged coherence time for both basal divacancies is the same, i.e., $T_{2}^{*}=0.7 \mu \mathrm{s}, T_{2}=1.4 \mathrm{~ms}$, which confirms that the large transverse ZFS is the main driving force for an increased coherence protection in the $h k-\mathrm{V}_{\mathrm{C}} \mathrm{V}_{\mathrm{Si}}$ divacancy.

Our results for the different basal divacancies show that by engineering high zero-field splitting either by selecting different defects, or applying the strain to the system [45], one can achieve a substantial increase in the coherence time.

\section{CONCLUSIONS}

Understanding the relation between the electronic structure of spin defects and their coherence properties is pivotal to optimizing the conditions for solid-state qubit applications. In this work, we carry out a series of calculations to investigate the effect of the nuclear spin bath at avoided crossings on the quantum dynamics of divacancies in SiC. We validate our results with measurements of Ramsey fringes and Hahn-echo coherence times and find excellent agreement between theory and experiments. Our work provides a robust strategy to uncover the effect of the interaction of nuclear spins on solid-state qubits' decoherence over a wide range of applied magnetic fields.

Applying charge depletion [37] to electrically improve coherence, we are able to experimentally isolate and elucidate the duality of the nuclear bath impact on clock transitions' dynamics. We discover that in the presence of strongly coupled nuclear spins, multiple clock transitions in the frequency spectrum of the spin qubit can emerge. We identify and characterize the nuclei with high hyperfine coupling in these systems; the initialization of these nuclear spins should allow one to achieve significantly higher coherence times under applied magnetic fields, while the nuclear-spin-dependent spectral features provide guidance for the development of a new class of electronnuclear two-qubit gates. We find that the effect of weakly coupled nuclear spins can be treated as a stochastic classical noise at the clock transition, and that the total amplitude of the coupling is a good descriptor of the coherence time. We further probe the classical-to-quantum transition of the noise and show how a tunable backaction of the electronic spin emerges with applied magnetic fields.
In summary, the joint computational and experimental study allows us to probe the nuclear environment around divacancies in $\mathrm{SiC}$ at avoided crossings down to the single nuclear spin level. The experimental validation of the predictions made here paves the way to optimize and eventually design the coherence properties of spin qubits yet to be explored experimentally.

\section{ACKNOWLEDGMENTS}

This work made use of resources provided by the University of Chicago's Research Computing Center, the UChicago MRSEC (NSF Grant No. DMR-1420709) and Pritzker Nanofabrication Facility, which receives support from the SHyNE, a node of the NSF's National Nanotechnology Coordinated Infrastructure (NSF Grant No. ECCS-1542205). M.O. and G.G. are supported by AFOSR FA9550-19-1-0358, H.M. by the UChicago MRSEC (NSF Grant No. DMR-1420709). K.C.M., J.P.B., C.P.A., A.B., and D.D.A. are supported by AFOSR FA9550-19-1-0358, DARPA D18AC00015KK1932, and ONR N00014-17-13026.

M.O. developed the model and conducted the dynamics calculations. H.M. performed the $a b$ initio density functional theory calculations. M.O., K.C.M., and J.P.B. designed the experiments. K.C.M. and J.P.B. performed the experiments. M.O., K.C.M., and J.P.B. analyzed the data. C.P.A. fabricated the sample. K.C.M. and J.P.B. developed the confocal microscope setup, with the assistance of A.B. D.D.A. advised on all experimental efforts. G.G. advised on theoretical efforts and supervised the project. All authors contributed to the writing of the manuscript.

\section{APPENDIX A: CONVERGENCE OF THE gCCE METHOD WITH RESPECT TO CLUSTER SIZE}

We applied the gCCE method with and without Monte Carlo bath state sampling to reveal the qubit dynamics at clock transition of the basal divacancy, observed in the Ramsey and Hahn-echo experiments. In Fig. 7 we show the time evolution of the off-diagonal element of the density matrix of the qubit for one random spatial configuration of nuclear spins.

We emphasize that considering mean-field effects of nuclear interactions is crucial in order to obtain the correct dynamics of the coherent state in the system studied here. The results without mean-field corrections (left panels of Fig. 7) may significantly underestimate the coherence on longer timescales, and high-order corrections may lead to divergence of the results.

We find that the decay of the observed central spin Ramsey envelope can be accurately described by performing calculations at the gCCE1 level with Monte Carlo bath state sampling [Fig. 7(a)]. Since gCCE1 simulations do not explicitly include nuclear-nuclear interactions, our results 


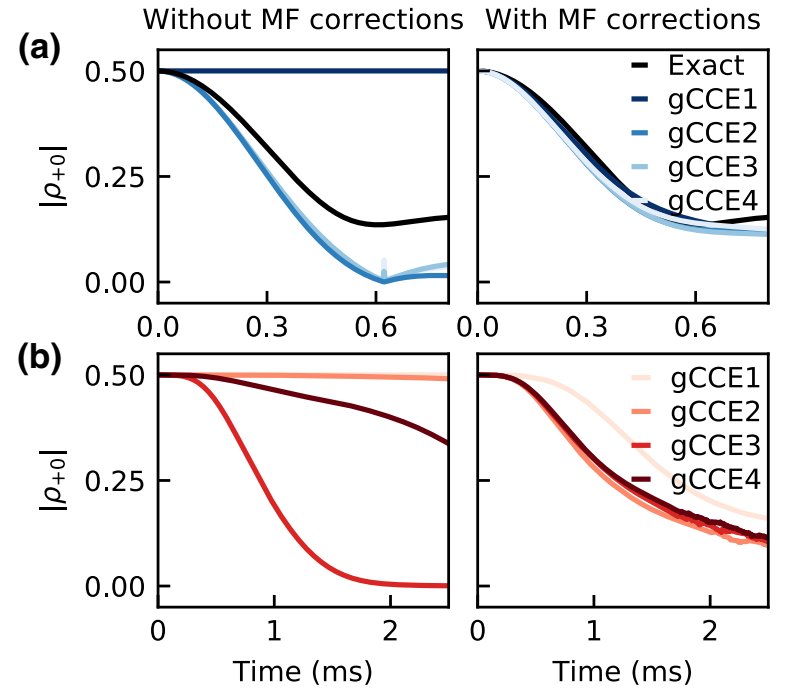

FIG. 7. Single defect coherence of the basal $k h$ divacancy in $4 H$-SiC predicted by different theoretical approximations. (a),(b) The absolute value of the off-diagonal elements of the density matrix $\left|\rho_{0+}\right|=|\langle 0|\hat{\rho}|+\rangle|$ for one random nuclear configuration corresponding to Ramsey (a) and Hahn-echo (b) experiments. The results of the gCCE at different orders (from 1 to 3 ) without mean-field (MF) interactions (left) and with mean-field corrections (right). The exact solution for a bath of nine nuclear spins for the Ramsey decay is shown as a black line.

suggest that in the Ramsey experiment the dominant decoherence mechanism is the static Overhauser field generated by nuclear spins, in agreement with Ref. [33].

On the other hand, we find that, for most configurations, gCCE2 is necessary and sufficient to converge the value of the Hahn-echo coherence [Fig. 7(b)] time, confirming the significant contribution of nuclear-nuclear interactions. This result differs from that of calculations for $\mathrm{Si}: \mathrm{Bi}$ [28] where convergence is achieved only with the inclusion of three-spin clusters. These differences suggest that accounting for the Overhauser field at the microscopic level, as proposed in our work, may effectively decrease the order necessary to converge the calculations presented in Ref. [28]; however, further in-depth study is necessary to reach a definitive conclusion.

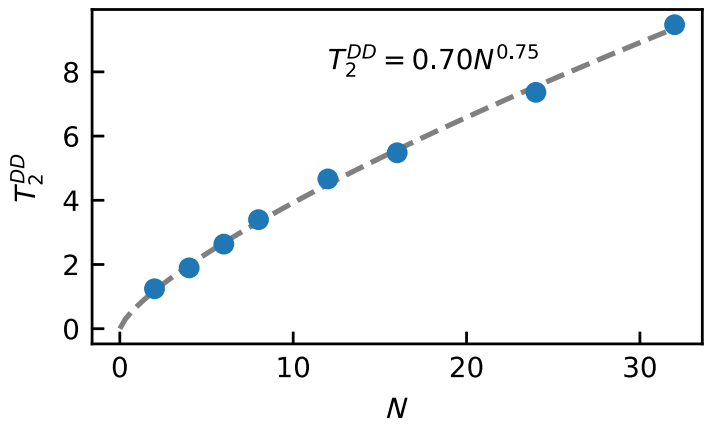

FIG. 9. Coherence time of the basal divacancy at zero magnetic field as a function of the number of decoupling pulses $N$.

Finally, we note that we observe the convergence of coherence times at avoided crossings at the gCCE2 level even for more complicated CPMG (Carr-PurcellMeiboom-Gill) sequences (Fig. 8), with the number of decoupling pulses $2 \leq N \leq 8$. A higher number of pulses requires the inclusion of higher-order cluster contributions. The coherence time $T_{2}^{D D}$ (Fig. 9) scales sublinearly in the considered number of pulses range.

\section{APPENDIX B: CALCULATION OF CLUSTER CONTRIBUTIONS IN THE gCCE}

The cluster contributions are defined recursively as

$$
\tilde{\rho}_{a b}^{\{C\}}=\frac{\left\langle a\left|\hat{\rho}_{C}(t)\right| b\right\rangle}{\prod_{C^{\prime}} \tilde{\rho}_{a b}^{\left\{C^{\prime} \subset C\right\}}}
$$

where $\rho_{C}(t)$ is the density matrix of cluster $C$ and the superscript $\left\{C^{\prime}\right\}$ indicates all subclusters of $C$, including the free central spin subcluster $\{0\}$. The density matrix $\rho_{C}(t)$ is computed using the time-ordered propagator

$$
\hat{U}_{C}(t)=\mathcal{T}\left[\exp \left(-i \int_{0}^{t} \hat{H}(\tau) d \tau\right)\right]
$$

where $\mathcal{T}$ is the time ordering operator and $\hat{H}(\tau)$ is the timedependent Hamiltonian, which includes only the interactions within a given cluster.

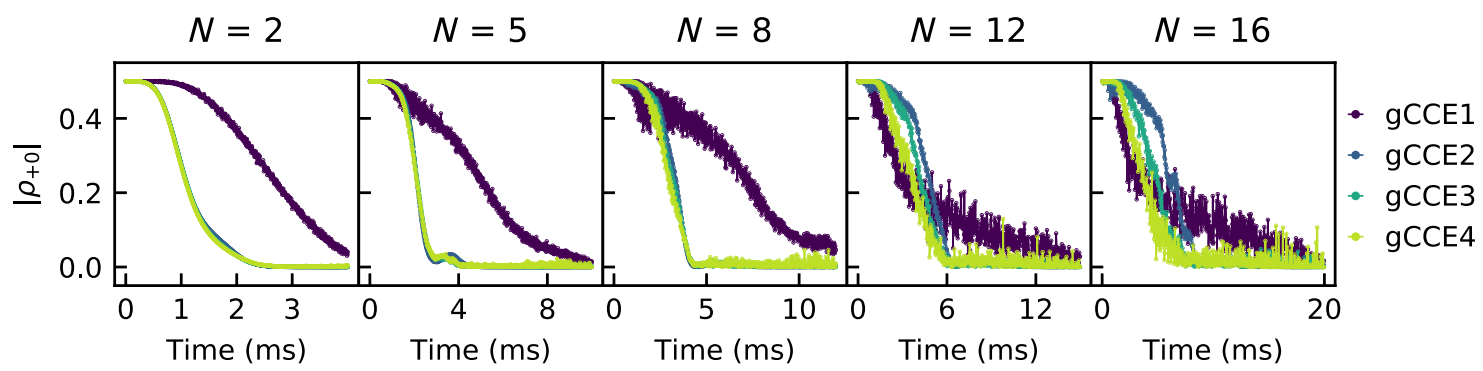

FIG. 8. Off-diagonal element of the density matrix of the qubit at zero magnetic field for various decoupling pulses $N$. 
In order to evaluate the density matrix $\rho_{C}(t)$ in Eq. (B1), we compute the evolution of the initial density matrix of a given cluster as

$$
\hat{\rho}_{C}(t)=\hat{U}_{C} \hat{\rho}_{C}(0) \hat{U}_{C}^{\dagger}
$$

using the time-ordered propagator $\hat{U}_{C}$ given in Eq. (B2). The Hamiltonian used to model Ramsey experiments does not depend on time $\hat{H}(\tau)=\hat{H}_{C}$, and the propagator is trivial:

$$
\hat{U}_{C}(t)=e^{-i \hat{H}_{C} t}
$$

The Hamiltonian $\hat{H}_{C}$ is equal to the system Hamiltonian (1), which contains only the central spin and a given cluster of nuclear spins:

$$
\hat{H}_{C}=\hat{H}_{e}+\sum_{i \subset C} \mathbf{S A I} \mathbf{I}_{i}-\sum_{i \subset C} \gamma_{n} B_{z} \hat{I}_{z, i}+\sum_{i \neq j \subset C} \mathbf{I}_{i} \mathbf{P} \mathbf{I}_{j} .
$$

Under the dynamical decoupling to the qubit, by assuming ideal instantaneous control pulses, we can write the propagator as

$$
\hat{U}_{C}(t)=\mathcal{T}\left[e^{-i \hat{H}_{C} \tau} e^{-i \sigma_{\{x, y, z\}} \phi / 2} e^{-i \hat{H}_{C} \tau}\right]^{N},
$$

where $\sigma_{\{x, y, z\}}$ is one of the Pauli matrices (depending on the type of pulse), spanned by two qubit levels, $\tau$ is the delay between pulses, $\phi$ is the angle of rotation (equal to $\pi$ for CPMG, XY4 sequences [46]; it may be varied to represent more complicated schemes [47]), and $N$ is number of pulses. For example, the propagator used to model Hahn-echo experiments with a $\pi$ rotation about the $x$ axis is defined as

$$
\hat{U}_{C}^{\mathrm{HE}}(t)=e^{-i \hat{H}_{C} \tau} e^{-i \sigma_{x} \pi / 2} e^{-i \hat{H}_{C} \tau}
$$

The Pauli matrices for qubit levels $|0\rangle$ and $|1\rangle$ are defined as

$$
\begin{aligned}
& \sigma_{x}=(|0\rangle\langle 1|+| 1\rangle\langle 0|), \\
& \sigma_{y}=i(|1\rangle\langle 0|-| 0\rangle\langle 1|), \\
& \sigma_{z}=(|0\rangle\langle 0|-| 1\rangle\langle 1|) .
\end{aligned}
$$

When using Monte Carlo sampling of the bath states, we perform the CCE calculations for each pure bath state separately. In the pure bath state, each nucleus is initialized in the spin-up or spin-down state, and in the mixed state each nuclear spin has a classical probability of being in one of the two states. We define the density matrix elements of the central spin as

$$
\hat{\rho}_{a b}(t)=\sum_{B} p_{J} \hat{\rho}_{a b}^{J}(t)
$$

where the elements of the density matrix $\hat{\rho}_{a b}$ are written as a summation over pure bath states $J$, with elements $\hat{\rho}_{a b}^{J}$ and probability $p_{J}$. In the case of a completely randomized bath (the density matrix of each nuclear spin is equal to $I / 2$ ), the probability $p_{J}$ is the same for all pure bath states. At the typical temperatures of the experiment (greater than or equal to $4 \mathrm{~K}$ ) the nuclear bath can be considered completely randomized.

The procedure used to evaluate the density matrix elements is the following. First, we generate a set of random pure bath states. For each bath state, we perform CCE calculations to obtain the electron spin density matrix. Finally, we compute the density matrix elements for the mixed bath state from Eq. (B11), and verify the convergence of density matrix elements $\rho_{a b}(t)$ with respect to the number of generated bath states (see below).

We add the mean-field effect of the bath spins outside a given cluster, by adding the $\hat{H}_{\mathrm{mf}}$ term into the cluster Hamiltonian (B5). The mean-field term is defined as

$$
\hat{H}_{\mathrm{mf}}=\sum_{i \not \subset C}\left[A_{z z}\left\langle I_{z, i}\right\rangle \hat{S}_{z}+\sum_{j \subset C} P_{z z}\left\langle I_{z, i}\right\rangle \hat{I}_{z, j}\right],
$$

where $\left\langle I_{z, i}\right\rangle= \pm \frac{1}{2}$, is the projection of the nuclear spin in the $z$ direction. The sign depends on the initial state of nuclei $i$ in the given random bath state.

For a specific bath state at the given time step, the contribution of some clusters can be very close to 0 , leading to the numerically unstable value of the density matrix elements. We identify such points when the elements of the density matrix are larger than 1 , and remove them from computing the average density matrix at the given time.

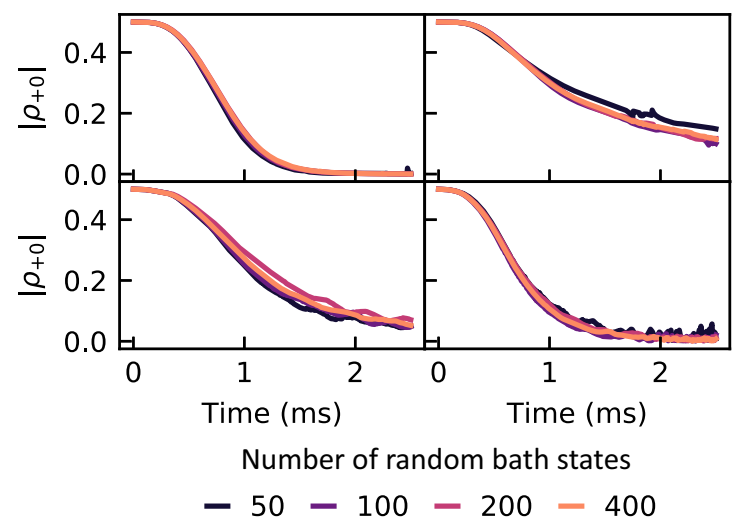

FIG. 10. The convergence of the off-diagonal element $\rho_{+0}$ of the density matrix in Hahn-echo measurements of the $k h-\mathrm{V}_{\mathrm{C}} \mathrm{V}_{\mathrm{Si}}$ divacancy as a function of the number of random bath states. 

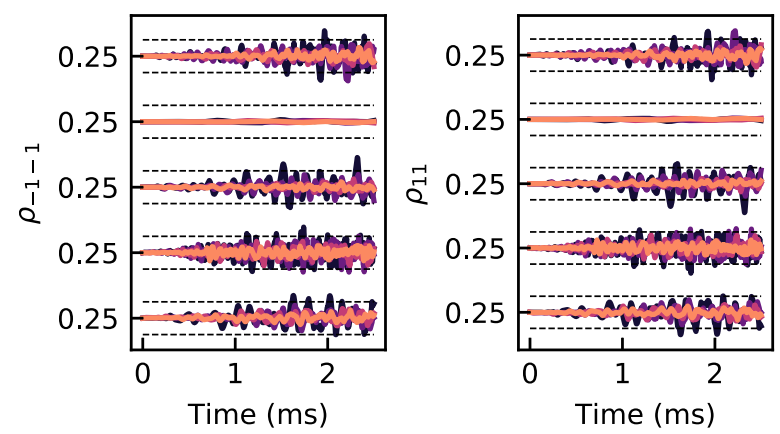

Number of random bath states

$$
-50-100-200-400
$$

FIG. 11. The convergence of diagonal elements $\rho_{-1-1}$ and $\rho_{11}$ of the density matrix in Hahn-echo measurements of the $k h$ $\mathrm{V}_{\mathrm{C}} \mathrm{V}_{\mathrm{Si}}$ divacancy as a function of the number of random bath states. Calculations are performed for five random nuclear spin configurations at zero magnetic field. Gray dashed lines correspond to the $\pm 10 \%$ range. We set $\rho_{00}=0.5$ for all calculations.

The off-diagonal element of the density matrix of the basal divacancy converges quickly with the number of random bath states (Fig. 10). On the other hand, the diagonal elements are predicted with a significant amount of noise (Fig. 11). The noise in computed diagonal elements due to the random states is more significant than the effect of other parameters. Therefore, we consider the changes in the population on the qualitative level, while we use off-diagonal elements for quantitative predictions of the entanglement with nuclear bath.

In the presence of the strongly coupled nuclear spins at the nonzero magnetic field, the results converge only when the exact averaging over the states of the strongly coupled spin is followed. In such systems, the modified sampling procedure can be implemented. For each state of the strongly coupled nuclear spins, we sample the same number of random bath states to obtain a density matrix element. The resulting matrix element for the randomized bath is an average of the elements, computed for each pure state of strongly coupled nuclear spins.

In computing the diagonal elements of the density matrix we renormalize the changing diagonal elements of the density matrix for each time step to ensure that the total population is equal to 1 .

\section{APPENDIX C: CALCULATIONS OF HYPERFINE COUPLING OF NUCLEAR SPINS}

The inhomogeneous coherence time $T_{2}^{*}$ is directly related to the hyperfine couplings. Under weak magnetic fields, the nuclear spin flips can be induced by both the hyperfine coupling and dipolar-dipolar interactions between nuclear spins [39]; hence, accurate predictions of the hyperfine parameters are necessary to correctly compute the Hahn-echo coherence time as well.

We perform $a b$ initio density functional theory (DFT) calculations to predict hyperfine coupling constants for nuclear spins at distances of up to $1 \mathrm{~nm}$ from the defect and we use the point dipole approximation for spins at larger distances. DFT calculations using the Perdew-Burke-Ernzerhof (PBE) functional are carried out with the GIPAW code [48] using single-particle wavefunctions obtained with the Quantum Espresso code [49]. Wavefunctions are represented on a planewave basis with a kinetic energy cutoff of 40 Ry. GIPAW pseudopotentials [50] are used to model electron-ion interactions. Divancancies are modeled with $9 \times 5 \times 2$ orthorhombic supercells containing 1438 atoms and the Brillouin zone is sampled with the $\Gamma$ point only.

We define a weakly coupled bath as a bath in which the nuclear spins do not change the energy splitting of the defect. We impose a cutoff of the hyperfine couplings of $A_{z z}<1 \mathrm{MHz}$ present in the weakly coupled bath, which is of typical order of magnitude compared to strongly coupled nuclear spins in the nitrogen-vacancy center [51,52]. The ensemble dynamics throughout the text is shown for the weakly coupled bath.

\section{APPENDIX D: PROPERTIES OF BASAL DIVACANCIES IN THE PRESENCE OF NUCLEAR SPINS}

\section{Impact of the magnetic field on the initial qubit state of basal divacancy}

For the nonzero magnetic field, the general qubit states are defined as $|+\rangle=a\left|1_{z}\right\rangle+b\left|-1_{z}\right\rangle$ and $|0\rangle=\left|0_{z}\right\rangle$. Amplitudes $a$ and $b$ are different for varied magnetic fields. For example, at $B_{z}=0 \mathrm{mT}$, the amplitudes are equal, $|a|=|b|=1 / \sqrt{2}$, and at $B_{z}=0.5 \mathrm{mT}$, the ratio between the amplitudes is $|a|:|b| \approx 2: 1$. In the presence of nuclear spins, when the qubit is prepared in the $|\psi\rangle$ state, the transition occurs for all possible bath states. For the weakly coupled nuclear bath, the Overhauser field for each bath state is compensated by randomization of the bath $\left(\sum_{n} A_{i z} I_{n} \approx 0, I_{n}= \pm \frac{1}{2}\right)$. Then, the interactions between the central qubit and nuclei do not impose significant changes in the qubit level structures compared to the isolated defect in the external magnetic field. This can be seen by detecting the evolution of the diagonal elements of the density matrix of the qubit (Fig. 12). Therefore, for the weakly coupled bath, we consider initial qubit states as eigenstates of $\hat{H}_{e}$.

In the presence of the strongly coupled nuclear spins, the Overhauser field from these nuclear spins cannot be compensated by other nuclei $\left(A_{i z}^{\text {strong }} \gg \sum_{\text {weak }} A_{i z}^{\text {weak }}\right)$, and can shift the qubit levels substantially.

As an example, we consider the most extreme case, when hyperfine coupling $A_{i z}=50.96 \mathrm{MHz}$ is higher than 


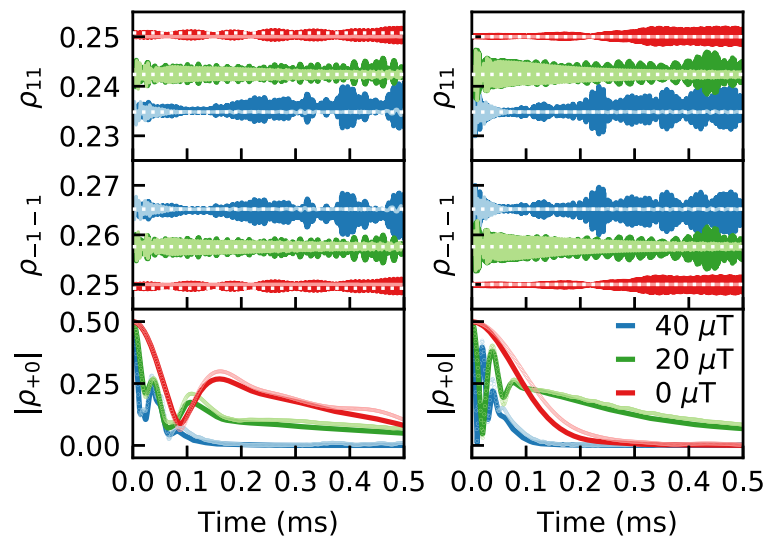

FIG. 12. Density matrix elements of the $k h-\mathrm{V}_{\mathrm{C}} \mathrm{V}_{\mathrm{Si}}$ divacancy for two random nuclear spin spatial configurations of a weakly coupled nuclear bath (in columns) as a function of the magnetic field. The lines with brighter color correspond to the exact solution for a smaller bath (nine nuclear spins). The dotted white line corresponds to the initial value of $\rho_{i i}$. We set $\rho_{00}=0.5$ for all calculations.

the transverse ZFS (Fig. 13). The initial nuclear spin states are completely randomized. The initial adiabatic change in the qubit $|+\rangle$ state occurs significantly faster than the following decoherence, and the rate of decoherence is mostly independent of the initial qubit state.

For the given nuclear spin bath, in both initial states of the qubit, the off-diagonal elements of the density matrix decay an order of magnitude slower at $0.87 \mathrm{mT}$ compared to $0.4 \mathrm{mT}$. This allows us to use the initial qubit state $|\psi\rangle$ with $|+\rangle=(1 / \sqrt{2})(|-1\rangle+|+1\rangle)$ for systems with
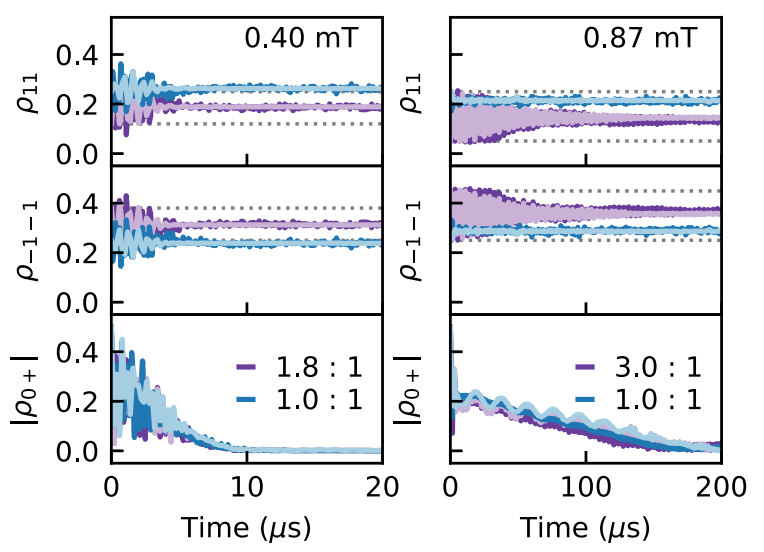

FIG. 13. Density matrix elements of the $k h-\mathrm{V}_{\mathrm{C}} \mathrm{V}_{\mathrm{Si}}$ divacancy for a nuclear configuration containing a strongly coupled nuclear spin with $A_{i z}=8.93 \mathrm{MHz}$ at low magnetic fields. The color indicates the ratio of $|a|$ and $|b|$ in the initial $|+\rangle=a\left|-1_{z}\right\rangle+b\left|+1_{z}\right\rangle$ state of the qubit. In each case, the qubit is initially prepared in the state $|\psi\rangle=(1 / \sqrt{2})(|+\rangle+|0\rangle)$. The lines with brighter color correspond to the exact solution with the same initial state for a smaller bath (nine nuclear spins). The dotted gray line corresponds to the initial value of the density matrix diagonal element. We set $\rho_{00}=0$ for all calculations.
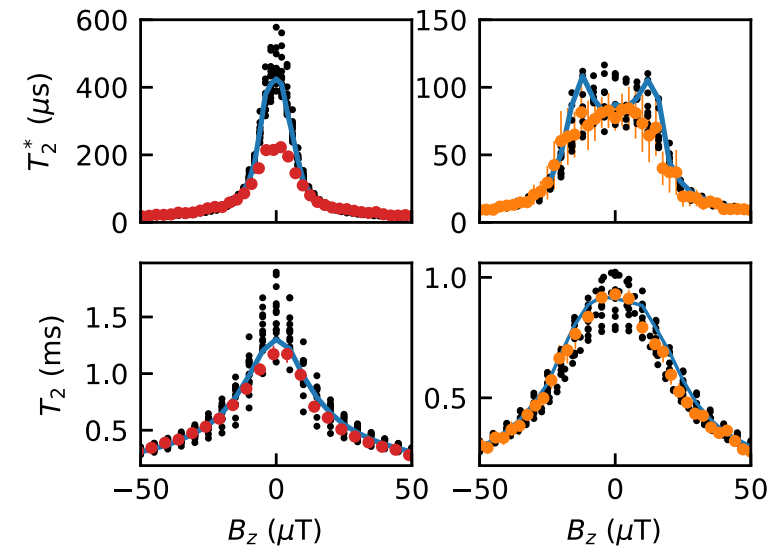

FIG. 14. The distribution of coherence times $T_{2}^{*}$ (top) and $T_{2}$ (bottom) for theoretical nuclear configurations obtained for VVA (red) and VVB (orange). Blue lines correspond to configurations shown in the main text.

strongly coupled nuclear spins, and allow it to evolve adiabatically even at relatively high magnetic fields to correctly predict Ramsey decoherence.

\section{Determining the experimental configurations}

To find the theoretical nuclear configurations that correspond to the experimentally measured basal divacancies, we apply the following procedure.

First, we generate a large set of random nuclear spin configurations. Out of this set, we identify those with or without strongly coupled nuclear spins. For each experimental divacancy, we determine the subsets of configurations with the same number of identifiable strongly coupled nuclear spins. For VVA, we determine a subset of random nuclear spin configurations with no identifiable strongly coupled nuclear spins; for VVB, configurations containing one strongly coupled nuclear spin with $A_{i z} \sim$ $0.6 \mathrm{MHz}$; and for VVC, configurations containing three strongly coupled nuclear spins with $\sum A_{i z} \sim 3 \mathrm{MHz}$.

For VVC, we identify the configurations containing six minima in the frequency spectrum. For VVA and VVB, we identify the nuclear configurations that have a similar value of $T_{2}^{*}$ at the applied magnetic field of $50 \mu \mathrm{T}$ to the corresponding experimental system. This value is instrumental to find the configurations with a similar total coupling of the bath and allows us to choose the configurations with the coupling of the nuclear bath to the electron spin closest to the experimental system. One such configuration for each divacancy is shown in the main text. All of the configurations for VVA and VVB are shown in Fig. 14.

\section{Initialization of strongly coupled nuclear spins}

We show the theoretical nuclear configuration with the strongly coupled nucleus of $A_{i z}=0.75 \mathrm{MHz}$ present, compared in the main text to VVB (Fig. 15), and study the effect of its initial state. If the strongly coupled nuclear spin 

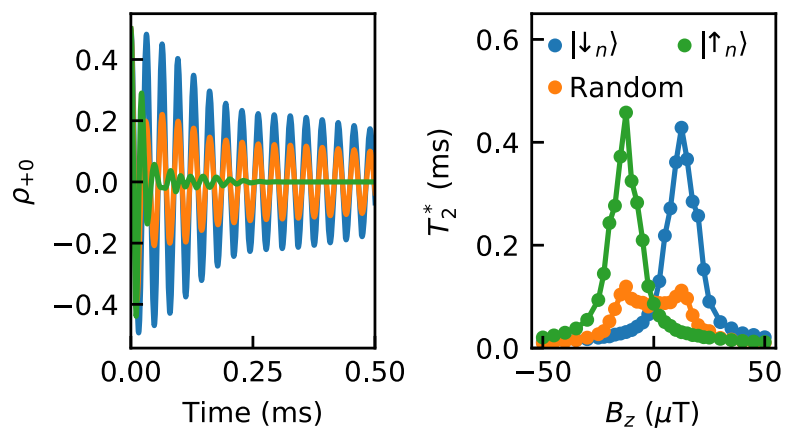

FIG. 15. Ramsey oscillations in the system with the strongly coupled nuclear spin. Left: $\rho_{0+}$ at the magnetic field of $12.5 \mu \mathrm{T}$ in the presence of the nuclear spin with $A_{i z}=0.75 \mathrm{MHz}$ initialized in the spin-up $\left(\left\langle\uparrow_{n}\right|\right.$, orange), spin-down $\left(\left\langle\downarrow_{n}\right|\right.$, blue), or thermalized (green) state. Right: $T_{2}^{*}$ against the magnetic field for a system with differently initialized strongly coupled nuclear spins. All other nuclear spins are completely randomized.

is initially in the thermalized state, the decay of the offdiagonal element contains two components: the fast initial decay due to the fast entanglement with the bath states, containing the strongly coupled nuclear spin parallel to the magnetic field, and the slow decay due to the entanglement with the bath states with strongly coupled spin down. When we initialize the strongly coupled nuclei in one specific state, only one mode of the decay is observed. The initialization of the strongly coupled nuclear spin antiparallel to the magnetic field (left panel of Fig. 15, blue line) significantly prolongs coherence, while the antiparallel orientation shortens the coherence time.

\section{Nuclear bath with strongly coupled nuclear spins}

Here, we theoretically study the coherence time of several additional configurations with strongly coupled nuclear spins. The coherence time of the basal divacancy can have several resonances as a function of the magnetic
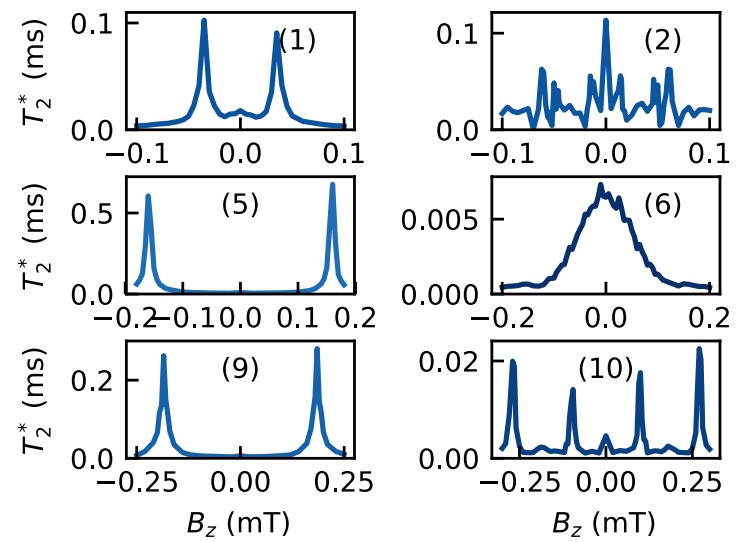

TABLE I. Highest five hyperfine couplings $A_{i z}$ of the configurations, shown in Fig. 16. The nuclear couplings giving rise to the peak splitting are highlighted in bold.

\begin{tabular}{cccccc}
\hline \hline No. & \multicolumn{5}{c}{$A_{i z}(\mathrm{MHz})$} \\
\hline 1 & $\mathbf{1 . 9 7}$ & 0.46 & 0.17 & 0.12 & 0.11 \\
2 & $\mathbf{2 . 0 9}$ & $\mathbf{1 . 3 4}$ & $\mathbf{0 . 4 8}$ & 0.06 & 0.04 \\
3 & $\mathbf{2 . 0 4}$ & $\mathbf{1 . 9 7}$ & $\mathbf{1 . 0 1}$ & 0.12 & 0.12 \\
4 & $\mathbf{4 . 1 9}$ & 0.09 & 0.04 & 0.04 & 0.04 \\
5 & $\mathbf{8 . 9 3}$ & 0.13 & 0.09 & 0.05 & 0.05 \\
6 & 2.04 & 1.97 & 1.93 & 1.21 & 0.72 \\
7 & $\mathbf{1 0 . 3 9}$ & 0.53 & 0.12 & 0.04 & 0.03 \\
8 & $\mathbf{1 0 . 3 9}$ & 0.46 & 0.13 & 0.09 & 0.09 \\
9 & $\mathbf{1 0 . 3 9}$ & 0.15 & 0.12 & 0.07 & 0.05 \\
10 & $\mathbf{1 0 . 3 9}$ & $\mathbf{4 . 8 3}$ & 0.15 & 0.11 & 0.07 \\
11 & $\mathbf{8 . 9 3}$ & $\mathbf{8 . 2 3}$ & $\mathbf{2 . 0 9}$ & 0.19 & 0.17 \\
12 & $\mathbf{5 0 . 9 5}$ & 0.09 & 0.07 & 0.04 & 0.03 \\
\hline \hline
\end{tabular}

field if strongly coupled nuclei are present. The main reason for the appearance of the maxima is due to completely negated interactions with the strongly coupled nuclear spins in spin-up or spin-down states.

When hyperfine couplings for several nuclei are significantly higher than the coupling to the remaining bath, the number of peaks can be greater than two (Fig. 16 and Table I). For each pure bath state, the resonance occurs at the magnetic field $B_{z}=\sum_{\text {strong }} \pm A_{i z} /\left(2 \gamma_{e}\right)$. The resulting coherence time dependence for the thermalized bath will show peaks either aligned with the peaks of pure bath states [Fig. 16(9)], or at the overlap of two peaks [Fig. 16(3)]. In the presence of many strongly coupled nuclear spins the peaks can become indistinguishable [Fig. 16(6)].

\section{Single defect coherence time at different transverse ZFS}

In Fig. 17 we show the coherence time of basal divacancy as a function of the transverse ZFS $E$. We can see that a single defect $T_{2}$ scales sublinearly with $E$, while $T_{2}^{*}$
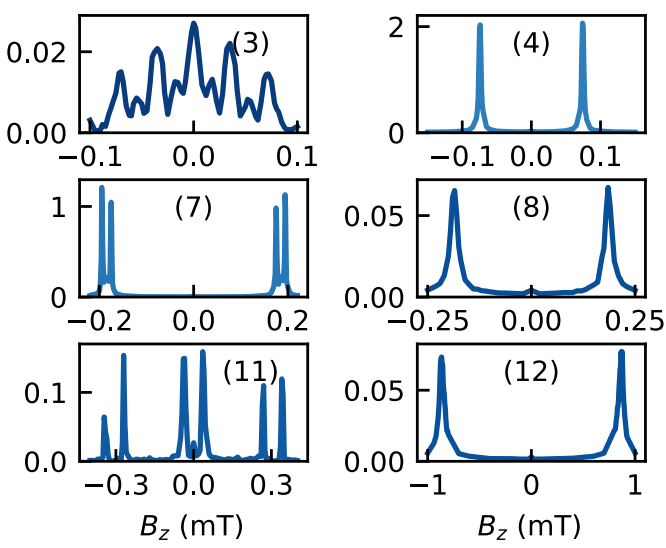

FIG. 16. Coherence time against the magnetic field for the theoretical configurations containing strongly coupled nuclear spins. Numbers (1-12) correspond to different bath configurations with one or several strongly coupled nuclear spins (Table I). 

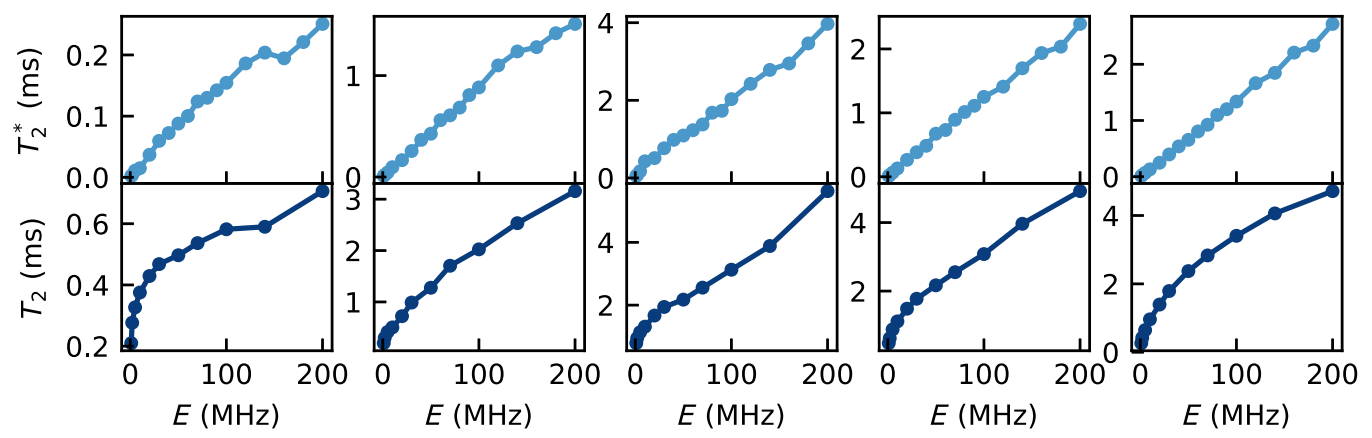

FIG. 17. Coherence time of the $k h-\mathrm{V}_{\mathrm{C}} \mathrm{V}_{\mathrm{Si}}$ divacancy for Ramsey (top) and Hahn-echo (bottom) experiments as a function of the transverse ZFS $E$ for five random nuclear spin configurations without strongly coupled nuclear spins. scales linearly. We note significant variability in values of the single defect coherence times at zero magnetic

\section{APPENDIX E: EXPERIMENTAL MEASUREMENTS OF THE $k h-V_{C} V_{S i}$ COHERENCE PROPERTIES}

Our $4 H$-SiC sample consists of a $20 \mu \mathrm{m}$ high-purity $i$ type $\mathrm{SiC}$ layer epitaxially grown on a $4^{\circ}$ off-axis miscut of the Si face of a high-purity semi-insulating SiC substrate (serial number A3177-14, Norstel AB). Neutral divacancies are uniformly produced throughout the epitaxial $i$-type $4 H$-SiC by electron irradiation with $2 \mathrm{MeV}$ electrons at a dose of $3 \times 10^{12} \mathrm{e} / \mathrm{cm}^{2}$ followed by annealing at $850{ }^{\circ} \mathrm{C}$ for $30 \mathrm{~min}$ in Ar. A coplanar capacitor structure with a $10 \mu \mathrm{m}$ gap width and a wire with $10 \mu \mathrm{m}$ width made of $\mathrm{Ti} / \mathrm{Au}$ are then patterned on the sample surface using electron beam lithography. Samples are cooled to 5 $\mathrm{K}$ in a closed-cycle cryostat (Cryostation s100, Montana Instruments).

The confocal microscope consists of a $905 \mathrm{~nm}$ excitation laser (QFLD-905-200S, QPhotonics) for off-resonant spin initialization, as well as a narrow-line tunable laser (DL pro, TOPTICA Photonics) for resonant spin readout. We focus these excitation beams through a microscope objective (LCPLN100XIR, Olympus). We detect the filtered optical signal with greater than $80 \%$ quantum efficiency using a low-jitter, low-dark count superconducting nanowire single-photon detector (SNSPD; Opus One, Quantum Opus). Electrical pulses from the SNSPD are counted using a data acquisition module (PCI-6259, National Instruments).

We drive the spin transition $|0\rangle \leftrightarrow|+\rangle$ using signal generators (SG396, Stanford Research Systems) modulated by an arbitrary waveform generator (HDAWG8, Zurich Instruments). The output of the signal generator is routed to the on-chip wire, which produces ac magnetic fields. Vector control of the magnetic field is obtained using a three-axis electromagnet outside the cryostat.

[1] E. D. Herbschleb, H. Kato, Y. Maruyama, T. Danjo, T. Makino, S. Yamasaki, I. Ohki, K. Hayashi, H. Morishita,
M. Fujiwara, and N. Mizuochi, Ultra-long coherence times amongst room-temperature solid-state spins, Nat. Commun. 10, 3766 (2019).

[2] L. Robledo, L. Childress, H. Bernien, B. Hensen, P. F. A. Alkemade, and R. Hanson, High-fidelity projective readout of a solid-state spin quantum register, Nature 477, 574 (2011).

[3] J. R. Weber, W. F. Koehl, J. B. Varley, A. Janotti, B. B. Buckley, C. G. Van de Walle, and D. D. Awschalom, Quantum computing with defects, Proc. Natl. Acad. Sci. 107, 8513 (2010).

[4] C. L. Degen, F. Reinhard, and P. Cappellaro, Quantum sensing, Rev. Mod. Phys. 89, 035002 (2017).

[5] H. Seo, A. L. Falk, P. V. Klimov, K. C. Miao, G. Galli, and D. D. Awschalom, Quantum decoherence dynamics of divacancy spins in silicon carbide, Nat. Commun. 7, 12935 (2016).

[6] A. M. Tyryshkin, S. Tojo, J. J. L. Morton, H. Riemann, N. V. Abrosimov, P. Becker, H.-J. Pohl, T. Schenkel, M. L. W. Thewalt, K. M. Itoh, and S. A. Lyon, Electron spin coherence exceeding seconds in high-purity silicon, Nat. Mater. 11, 143 (2012).

[7] R. E. George, J. P. Edwards, and A. Ardavan, Coherent Spin Control by Electrical Manipulation of the Magnetic Anisotropy, Phys. Rev. Lett. 110, 027601 (2013).

[8] W. M. Witzel and S. Das Sarma, Quantum theory for electron spin decoherence induced by nuclear spin dynamics in semiconductor quantum computer architectures: Spectral diffusion of localized electron spins in the nuclear solid-state environment, Phys. Rev. B 74, 035322 (2006).

[9] M. H. Abobeih, J. Cramer, M. A. Bakker, N. Kalb, M. Markham, D. J. Twitchen, and T. H. Taminiau, Onesecond coherence for a single electron spin coupled to a multi-qubit nuclear-spin environment, Nat. Commun. 9, 2552 (2018).

[10] T. van der Sar, Z. H. Wang, M. S. Blok, H. Bernien, T. H. Taminiau, D. M. Toyli, D. A. Lidar, D. D. Awschalom, R. Hanson, and V. V. Dobrovitski, Decoherence-protected quantum gates for a hybrid solidstate spin register, Nature 484, 82 (2012).

[11] A. Reiserer, N. Kalb, M. S. Blok, K. J. M. van Bemmelen, T. H. Taminiau, R. Hanson, D. J. Twitchen, and M. Markham, Robust Quantum-Network Memory Using Decoherence-Protected Subspaces of Nuclear Spins, Phys. Rev. X 6, 021040 (2016).

[12] A. Bourassa, C. P. Anderson, K. C. Miao, M. Onizhuk, H. Ma, A. L. Crook, H. Abe, J. Ul-Hassan, T. Ohshima, N. T. Son, G. Galli, and D. D. Awschalom, Entanglement and 
control of single nuclear spins in isotopically engineered silicon carbide, Nat. Mater. 19, 1319 (2020).

[13] G. Wolfowicz, A. M. Tyryshkin, R. E. George, H. Riemann, N. V. Abrosimov, P. Becker, H.-J. Pohl, M. L. W. Thewalt, S. A. Lyon, and J. J. L. Morton, Atomic clock transitions in silicon-based spin qubits, Nat. Nanotechnol. 8, 561 (2013).

[14] M. H. Mohammady, G. W. Morley, and T. S. Monteiro, Bismuth Qubits in Silicon: The Role of epr Cancellation Resonances, Phys. Rev. Lett. 105, 067602 (2010).

[15] K. C. Miao, J. P. Blanton, C. P. Anderson, A. Bourassa, A. L. Crook, G. Wolfowicz, H. Abe, T. Ohshima, and D. D. Awschalom, Universal coherence protection in a solid-state spin qubit, Science 369, 1493 (2020).

[16] V. Ivády, Longitudinal spin relaxation model applied to point-defect qubit systems, Phys. Rev. B 101, 155203 (2020).

[17] A. Jarmola, V. M. Acosta, K. Jensen, S. Chemerisov, and D. Budker, Temperature- and Magnetic-Field-Dependent Longitudinal Spin Relaxation in Nitrogen-Vacancy Ensembles in Diamond, Phys. Rev. Lett. 108, 197601 (2012).

[18] W. F. Koehl, B. B. Buckley, F. J. Heremans, G. Calusine, and D. D. Awschalom, Room temperature coherent control of defect spin qubits in silicon carbide, Nature 479, 84 (2011).

[19] P. V. Klimov, A. L. Falk, D. J. Christle, V. V. Dobrovitski, and D. D. Awschalom, Quantum entanglement at ambient conditions in a macroscopic solid-state spin ensemble, Sci. Adv. 1, e1501015 (2015).

[20] N. T. Son, C. P. Anderson, A. Bourassa, K. C. Miao, C. Babin, M. Widmann, M. Niethammer, J. Ul Hassan, N. Morioka, I. G. Ivanov, F. Kaiser, J. Wrachtrup, and D. D. Awschalom, Developing silicon carbide for quantum spintronics, Appl. Phys. Lett. 116, 190501 (2020).

[21] D. J. Christle, A. L. Falk, P. Andrich, P. V. Klimov, J. U. Hassan, N. T. Son, E. Janzén, T. Ohshima, and D. D. Awschalom, Isolated electron spins in silicon carbide with millisecond coherence times, Nat. Mater. 14, 160 (2015).

[22] A. L. Crook, C. P. Anderson, K. C. Miao, A. Bourassa, H. Lee, S. L. Bayliss, D. O. Bracher, X. Zhang, H. Abe, T. Ohshima, E. L. Hu, and D. D. Awschalom, Purcell enhancement of a single silicon carbide color center with coherent spin control, Nano Lett. 20, 3427 (2020).

[23] S. J. Whiteley, G. Wolfowicz, C. P. Anderson, A. Bourassa, H. Ma, M. Ye, G. Koolstra, K. J. Satzinger, M. V. Holt, F. J. Heremans, A. N. Cleland, D. I. Schuster, G. Galli, and D. D. Awschalom, Spin-phonon interactions in silicon carbide addressed by gaussian acoustics, Nat. Phys. 15, 490 (2019).

[24] K. C. Miao, A. Bourassa, C. P. Anderson, S. J. Whiteley, A. L. Crook, S. L. Bayliss, G. Wolfowicz, G. Thiering, P. Udvarhelyi, V. Ivády, H. Abe, T. Ohshima, Á. Gali, and D. D. Awschalom, Electrically driven optical interferometry with spins in silicon carbide, Sci. Adv. 5, eaay0527 (2019).

[25] W. Yang and R.-B. Liu, Quantum many-body theory of qubit decoherence in a finite-size spin bath, Phys. Rev. B 78, 085315 (2008).

[26] M. Ye, H. Seo, and G. Galli, Spin coherence in twodimensional materials, npj Comput. Mater. 5, 44 (2019).
[27] W. Yang, W.-L. Ma, and R.-B. Liu, Quantum manybody theory for electron spin decoherence in nanoscale nuclear spin baths, Rep. Prog. Phys. 80, 016001 (2016).

[28] S. J. Balian, R.-B. Liu, and T. S. Monteiro, Keeping a spin qubit alive in natural silicon: Comparing optimal working points and dynamical decoupling, Phys. Rev. B 91, 245416 (2015).

[29] S. J. Balian, G. Wolfowicz, J. J. L. Morton, and T. S. Monteiro, Quantum-bath-driven decoherence of mixed spin systems, Phys. Rev. B 89, 045403 (2014).

[30] Z.-S. Yang, Y.-X. Wang, M.-J. Tao, W. Yang, M. Zhang, Q. Ai, and F.-G. Deng, Longitudinal relaxation of a nitrogen-vacancy center in a spin bath by generalized cluster-correlation expansion method, Ann. Phys. 413, 168063 (2020).

[31] W.-L. Ma, G. Wolfowicz, N. Zhao, S.-S. Li, J. J. Morton, and R.-B. Liu, Uncovering many-body correlations in nanoscale nuclear spin baths by central spin decoherence, Nat. Commun. 5, 4822 (2014).

[32] W.-L. Ma, G. Wolfowicz, S.-S. Li, J. J. L. Morton, and R.-B. Liu, Classical nature of nuclear spin noise near clock transitions of bi donors in silicon, Phys. Rev. B 92, 161403 (2015).

[33] G.-L. Zhang, W.-L. Ma, and R.-B. Liu, Cluster correlation expansion for studying decoherence of clock transitions in spin baths, Phys. Rev. B 102, 245303 (2020).

[34] W. M. Witzel, M. S. Carroll, L. Cywiński, and S. Das Sarma, Quantum decoherence of the central spin in a sparse system of dipolar coupled spins, Phys. Rev. B 86, 035452 (2012).

[35] A. L. Falk, B. B. Buckley, G. Calusine, W. F. Koehl, V. V. Dobrovitski, A. Politi, C. A. Zorman, P. X.-L. Feng, and D. D. Awschalom, Polytype control of spin qubits in silicon carbide, Nat. Commun. 4, 1819 (2013).

[36] P. Jamonneau, M. Lesik, J. P. Tetienne, I. Alvizu, L. Mayer, A. Dréau, S. Kosen, J.-F. Roch, S. Pezzagna, J. Meijer, T. Teraji, Y. Kubo, P. Bertet, J. R. Maze, and V. Jacques, Competition between electric field and magnetic field noise in the decoherence of a single spin in diamond, Phys. Rev. B 93, 024305 (2016).

[37] C. P. Anderson, A. Bourassa, K. C. Miao, G. Wolfowicz, P. J. Mintun, A. L. Crook, H. Abe, J. Ul Hassan, N. T. Son, T. Ohshima, and D. D. Awschalom, Electrical and optical control of single spins integrated in scalable semiconductor devices, Science 366, 1225 (2019).

[38] G. Wolfowicz, C. P. Anderson, A. L. Yeats, S. J. Whiteley, J. Niklas, O. G. Poluektov, F. J. Heremans, and D. D. Awschalom, Optical charge state control of spin defects in 4h-sic, Nat. Commun. 8, 1876 (2017).

[39] N. Zhao, S.-W. Ho, and R.-B. Liu, Decoherence and dynamical decoupling control of nitrogen vacancy center electron spins in nuclear spin baths, Phys. Rev. B 85, 115303 (2012).

[40] I. A. Merkulov, A. L. Efros, and M. Rosen, Electron spin relaxation by nuclei in semiconductor quantum dots, Phys. Rev. B 65, 205309 (2002).

[41] B. Gu and I. Franco, When can quantum decoherence be mimicked by classical noise?, J. Chem. Phys. 151, 014109 (2019). 
[42] D. Stanek, C. Raas, and G. S. Uhrig, From quantummechanical to classical dynamics in the central-spin model, Phys. Rev. B 90, 064301 (2014).

[43] P. Huang, X. Kong, N. Zhao, F. Shi, P. Wang, X. Rong, R.-B. Liu, and J. Du, Observation of an anomalous decoherence effect in a quantum bath at room temperature, Nat. Commun. 2, 570 (2011).

[44] L.-P. Yang, C. Burk, M. Widmann, S.-Y. Lee, J. Wrachtrup, and N. Zhao, Electron spin decoherence in silicon carbide nuclear spin bath, Phys. Rev. B 90, 241203 (2014).

[45] A. L. Falk, P. V. Klimov, B. B. Buckley, V. Ivády, I. A. Abrikosov, G. Calusine, W. F. Koehl, A. Gali, and D. D. Awschalom, Electrically and Mechanically Tunable Electron Spins in Silicon Carbide Color Centers, Phys. Rev. Lett. 112, 187601 (2014).

[46] G. de Lange, Z. H. Wang, D. Ristè, V. V. Dobrovitski, and R. Hanson, Universal dynamical decoupling of a single solid-state spin from a spin bath, Science 330, 60 (2010).
[47] S. S. Hegde, J. Zhang, and D. Suter, Efficient Quantum Gates for Individual Nuclear Spin Qubits by Indirect Control, Phys. Rev. Lett. 124, 220501 (2020).

[48] https://github.com/dceresoli/qe-gipaw (Accessed: July 14, 2020).

[49] P. Giannozzi et al., Quantum espresso: A modular and open-source software project for quantum simulations of materials, J. Phys.: Condens. Matter 21, 395502 (2009).

[50] https://sites.google.com/site/dceresoli/pseudopotentials (Accessed: July 14, 2020).

[51] J. Yun, K. Kim, and D. Kim, Strong polarization of individual nuclear spins weakly coupled to nitrogen-vacancy color centers in diamond, New J. Phys. 21, 093065 (2019).

[52] A. Dréau, J.-R. Maze, M. Lesik, J.-F. Roch, and V. Jacques, High-resolution spectroscopy of single nv defects coupled with nearby ${ }^{13} \mathrm{c}$ nuclear spins in diamond, Phys. Rev. B 85, 134107 (2012). 